\title{
GENERIC DIFFEOMORPHISMS WITH SUPEREXPONENTIAL GROWTH OF NUMBER OF PERIODIC ORBITS.
}

\author{
VADIM YU. KALOSHIN
}

Dedicated to the memory of my grandfather Meyer Levich

\section{INTRODUCTION}

Let $C^{r}(M, M)$ be the space of $C^{r}$ mappings of a compact manifold $M$ into itself with the uniform $C^{r}$-topology and $\operatorname{Diff}^{r}(M)$ be the space of $C^{r}$ diffeomorphisms of $M$ with the same topology. It is well-known that $\operatorname{Diff}^{r}(M)$ is an open subset of $C^{r}(M, M)$. For a map $f \in C^{r}(M)$, consider the number of isolated periodic points of period $n$ (i.e. the number of isolated fixed points of $f^{n}$ )

$$
P_{n}(f)=\#\left\{\text { isolated } x \in M: x=f^{n}(x)\right\} .
$$

In 1965 Artin \& Mazur [AM] showed that: there exists a dense set $\mathcal{D}$ in $C^{r}(M, M)$ such that for any map $f \in \mathcal{D}$ the number $P_{n}(f)$ grows at most exponentially with $n$, i.e. for some number $C>0$

$$
P_{n}(f) \leq \exp (C n) \text { for all } n \in \mathbb{Z}_{+} .
$$

Notice that the Artin-Mazur Theorem does not exclude the possibility that a mapping $f$ in $\mathcal{D}$ has a curve of periodic points $\gamma$, i.e. $\forall x \in \gamma, f^{n}(x)=x$ for some $n \in \mathbb{Z}_{+}$, because in this case $\gamma$ consists of nonisolated periodic points of period $n$ (see the last part of Theorem 3 3 for this nonisolated case).

Definition 1. We call a mapping (resp. diffeomorphism) $f \in C^{r}(M, M)$ (resp. $f \in$ $\operatorname{Diffr}^{r}(M)$ ) an Artin-Mazur mapping (resp. diffeomorphism) or simply A-M mapping (resp. diffeomorphism) if $P_{n}(f)$ grows at most exponentially fast.

Artin-Mazur [AM posed the following problem: what can be said about the set of $A-M$ mappings with only transversal periodic orbits in the space of $C^{r}$ mappings? Recall that a periodic orbit of period $n$ is called transversal if the linearization $d f^{n}$ at this point has for an eigenvalue no $n$-th roots of unity. Notice that a hyperbolic periodic point is always transversal, but not vice versa.

In what follows we consider not the whole space $C^{r}(M, M)$ of mappings of $M$ into itself, but only its open subset $\operatorname{Diff}^{r}(M)$. The first main result of this paper is an answer to ArtinMazur's question for the space of diffeomorphisms and a simple proof of it.

Theorem 1. Let $1 \leq r<\infty$. Then the set of $A$-M diffeomorphisms with only hyperbolic periodic orbits is dense in the space $\operatorname{Diff}^{r}(M)$ with the uniform $C^{r}$ topology.

Date: January 24, 1999. 
In a weak form this theorem says that A-M diffeomorphisms which satisfy the Kupka-Smale condition form a dense set in $\operatorname{Diff}^{r}(M)$. Recall that a diffeomorphism is called a Kupka-Smale (or $K$-S) diffeomorphism) if all its periodic points are hyperbolic and all associated stable and unstable manifolds intersect one another transversally. The Kupka-Smale theorem says that K-S diffeomorphisms form a residual set (see e.g. [PM]). The natural question is whether intersection of $\mathrm{A}-\mathrm{M}$ and $\mathrm{K}-\mathrm{S}$ diffeomorphisms can be dense in $\operatorname{Diff}^{r}(M)$. The answer is not easy, because methods of the proof of both theorems are of completely different nature and can not be applied simultaneously. If one omits the condition on transversality of stable and unstable manifolds, then Theorem 1 says that the intersection of A-M and K-S diffeomorphisms is dense.

A residual set in a finite-dimensional space can have Lebesgue measure zero. Therefore, the Kupka-Smale theorem does not imply that "almost every" diffeomorphism is a K-S diffeomorphism. In loose terms, a set $P \subset \operatorname{Diff}^{r}(M)$ is called prevalent (or "has a full measure") if for a generic finite-parameter family $\left\{f_{\epsilon}\right\}_{\epsilon \in \text { Ball }}$, the property $f_{\epsilon} \in P$ holds for almost every parameter value. In [K] it is proven that K-S diffeomorphisms form a prevalent set or have "a full measure". Finally, for a discussion of prevalence see section 4 .

In [AM Artin-Mazur also introduced the dynamical $\zeta_{f}$-function defined by

$$
\zeta_{f}(z)=\exp \left(\sum_{n=1}^{\infty} P_{n}(f) \frac{z^{n}}{n}\right) .
$$

For A-M diffeomorphisms the dynamical $\zeta_{f}$-function is analytic in some disk centered at zero. It is well-known that the dynamical $\zeta_{f}$-function of a diffeomorphism $f$ satisfying Axiom A has an analytic continuation to a rational function (e.g. $\mathrm{Ba}$ ).

In 1967 Smale [S] posed the following question (Problem 4.5, p.765):

Is the dynamical $\zeta_{f}$-function generically rational (i.e. is $\zeta_{f}$ rational for a residual set of $\left.f \in \operatorname{Diffr}^{r}(M)\right)$ ?

In [Si] it is shown that for the 3 -dimensional torus the $\zeta_{f}$-function is not rational. It turns out that for manifolds of dimension greater or equal than 2 it is not even analytic in any neighborhood of zero (see Theorem 3 below). Recall that a subset of a topological space is called residual if it contains a countable intersection of open dense subsets. We call a residual set a topologically generic set.

Finally, in 1978 R. Bowen asked the following question in his book [Bo]:

Let $h(f)$ denote the topological entropy of $f$. Does

$$
h(f)=\limsup _{n \rightarrow \infty} \log P_{n}(f) / n
$$

for a generic set of diffeomorphisms with respect to the $C^{r}$ topology?

It turns out the two above questions can be answered simultaneously for $C^{r}$ diffeomorphisms with $2 \leq r<\infty$. The second result is the following:

Theorem 2. Let $2 \leq r<\infty$. Then the set of $A-M$ diffeomorphisms is not topologically generic in the space of $C^{r}$ diffeomorphisms $\operatorname{Diff}^{r}(M)$ with the uniform $C^{r}$ topology.

We have the following consequences:

Corollary 1. The property of having a convergent $\zeta_{f}(z)$ function is not $C^{r}$-generic, nor is the equation $h(f)=\lim \sup _{n \rightarrow \infty} \log P_{n}(f) / n$. 
The first part is easy. To prove the second, notice that the topological entropy for any $C^{r}(r \geq 1)$ diffeomorphism $f$ of a compact manifold is always finite (see e.g. [HK]). Define the rate of growth of the number of periodic orbits by $\lim \sup _{n \rightarrow \infty} \log P_{n}(f) / n$. Then for diffeomorphisms which are not A-M diffeomorphisms, the rate of growth is always equal to infinity.

Since, an Axiom A diffeomorphism is an A-M diffeomorphism, we need to analyze the complement to Axiom A diffeomorphisms in the space of $\operatorname{Diff}^{r}(M)$. An example of a diffeomorphism with an arbitrarily fast growing number of periodic orbits is given in [RG]. Now we describe a "bad" domain, where the A-M property fails to be topologically generic.

In 1970 Newhouse found a domain in the space of $C^{r}$ diffeomorphisms $\operatorname{Diff}^{r}(M)$, where diffeomorphisms exhibiting homoclinic tangencies are dense $\mathbb{N}$. Such a domain is called $a$ Newhouse domain $\mathcal{N} \subset \operatorname{Diff}^{r}(M)$. Our third and main result is as follows:

Theorem 3. Let $2 \leq r<\infty$. Let $\mathcal{N} \subset \operatorname{Diff}^{r}(M)$ be a Newhouse domain. Then for an arbitrary sequence of positive integers $\left\{a_{n}\right\}_{n=1}^{\infty}$ there exists a residual set $\mathcal{R}_{a} \subset \mathcal{N}$, depending on the sequence $\left\{a_{n}\right\}_{n=1}^{\infty}$, with the property that $f \in \mathcal{R}_{a}$ implies that

$$
\limsup _{n \rightarrow \infty} P_{n}(f) / a_{n}=\infty \text {. }
$$

Moreover, there is a dense set $\mathcal{D}$ in $\mathcal{N}$ such that any diffeomorphism $f \in F$ has a curve of periodic points.

Let us note that Theorems 2 and 3 follow from a Theorem of Gonchenko-Shilnikov-Turaev which will be discussed in section 2 .

In such a domain Newhouse exhibited a residual set of diffeomorphisms with infinitely many distinct sinks $\mathbb{N}],[\mathbb{R}]$, and $[\mathbb{P T}]$. Now it is known as Newhouse's phenomenon. In a way Theorem 3 is similar to Newhouse's phenomenon in the sense that for a residual set a "bad" property holds true.

Continuing, Theorem 2 is a corollary of the first part of Theorem 3 . To see this fix the sequence $a_{n}=n^{n}$ and denote by $\mathcal{R}_{a}$ a set from Theorem 3 corresponding to this sequence. Assume that A-M diffeomorphisms form a residual set, then this set must intersect with $\mathcal{R}_{a}$ which is a contradiction.

It seems that based on Newhouse's phenomenon in the space $\operatorname{Diff}^{1}(M)$ with the $C^{1}$ topology, where $\operatorname{dim} M \geq 3$, found by Bonnati \& Diaz BD one can extend Theorems 2 and 3 to the case $r=1$ and $\operatorname{dim} M \geq 3$. The problem with this straightforward generalization is that the proof of the Gonchenko-Shilnikov-Turaev (GST) theorem is essentially two-dimensional. To generalize the GST theorem to the three-dimensional case one needs either to find an invariant two-dimensional surface and use the two-dimensional proof or find another proof. In personal communications, Lorenzo Diaz has shown to the author that an invariant two-dimensional surface can be constructed using the method from [BD. However, this extension is not straightforward and will appear separately from this paper.

Analogs of Theorems 2 and 3 can be formulated for the case of vector fields on a compact manifold of dimension at least 3. Reduction from the case of diffeomorphisms to the case of vector fields can be done using the standard suspension of a vector field over a diffeomorphism [PM.

1.1. Newhouse phenomenon and Palis conjecture. Newhouse showed that a Newhouse domain exists under the following hypothesis: 
Let a diffeomorphism $f \in \operatorname{Diff}^{r}(M)$ have a saddle periodic orbit $p$. Suppose stable $W^{s}(p)$ and unstable $W^{u}(p)$ manifolds of $p$ have a quadratic tangency. Such a diffeomorphism $f$ is called a diffeomorphism exhibiting a homoclinic tangency. Then arbitrarily $C^{r}$-close to $f$ in $\operatorname{Diff}^{r}(M)$ there exists a Newhouse domain. In particular, it means that by a small $C^{r}$ perturbation of a diffeomorphism $f$ with a homoclinic tangency one can generate arbitrarily quick growth of the number of periodic orbits.

On this account we would also like to mention the following conjecture, which is due to Palis [PT], about the space of diffeomorphisms of 2-dimensional manifolds:

Conjecture If $\operatorname{dim} M=2$, then every diffeomorphism $f \in \operatorname{Diff}^{r}(M)$ can be approximated by a diffeomorphism which is either hyperbolic or exhibits a homoclinic tangency.

This conjecture is proven for approximations in the $C^{1}$ topology $[\overline{\mathrm{PS}}$. If this conjecture is true, then in the complement to the set of hyperbolic diffeomorphisms those diffeomorphisms with arbitrarily quick growth of number of periodic orbits form a topologically generic set.

Unfolding of homoclinic tangencies is far from being understood. In GST the authors describe the following important result: there does not exist a finite number of parameters to describe all bifurcations occurring next to a homoclinic tangency (see section 2, Corollary 2 for details). This implies that the complete description of bifurcations of diffeomorphisms with a homoclinic tangency is impossible.

This paper is organized as follows. In section 2.1 we state the Gonchenko-Shilnikov-Turaev result and give a proof of it in sections 2.2-2.5. Section 2.6 is devoted to the proof of Theorem 3 in the case $\operatorname{dim} M=2$. Reduction from Theorem 3 in the case $\operatorname{dim} M=2$ to Theorem 3 to the general case $\operatorname{dim} M \geq 2$ is done in section 2.7. Theorem 1 is proven in part 3. Discussion of open questions related to the main results is in section 4.

From now on we consider diffeomorphisms of a 2-dimensional compact manifold $M$. The general case when $\operatorname{dim} M \geq 2$ can be reduced to the 2-dimensional case by the standard suspension and the Fenichel theorem [F] (see section 2.7).

\section{Degenerate periodic orbits in a Newhouse domain and the Gonchenko-Shilnikov-Turaev Theorem GST}

Assume that a $C^{r}$ diffeomorphism $f$ exhibits a homoclinic tangency. By the Newhouse theorem $\mathbb{N}$, in each $C^{r}$ neighborhood of a diffeomorphism $f$ exhibiting a homoclinic tangency there exists a Newhouse domain.

Let us define a degenerate periodic point of order $k$ or a $k$-degenerate periodic point. Sometimes, it is also called a saddlenode periodic orbit of multiplicity $k+1$.

Definition 2. Let $f$ be a $C^{s}$ diffeomorphism of a 2-dimensional manifold having a periodic orbit $p$ of period $m$. A periodic point $p$ is called $k$-degenerate, where $k<s$, if the linear part of $f^{m}$ at point $p$ has a multiplier $\nu=1$ while the other multiplier is different in absolute value from the unit and a restriction of $f$ to the central manifold in some coordinate system can be written in the form

$$
x \mapsto x+l_{k+1} x^{k+1}+o\left(x^{k+1}\right) .
$$

Let $s>r$. Then $C^{s}$ diffeomorphisms are dense in the space $\operatorname{Diff}^{r}(M)$ and, therefore, in any Newhouse domain $\mathcal{N} \subset \operatorname{Diff}^{r}(M)$ (see e.g. $[\mathbb{P M}$ ). 
Theorem 4. (Theorem 4, GST) For any positive integers $s>k \geq r$ the set of $C^{s}$ diffeomorphisms having a $k$-degenerate periodic orbit is dense in a Newhouse domain $\mathcal{N} \subset$ $\operatorname{Diff}^{r}(M)$.

This theorem and Newhouse's theorem imply the following important result:

Corollary 2. [GST Let $f \in \operatorname{Diff}^{r}(M)$ be a diffeomorphism exhibiting a homoclinic tangency. There is no finite number s such that a generic s-parameter family $\left\{f_{\varepsilon}\right\}$ unfolding a diffeomorphism $f_{0}=f$ is a versal family of $f_{0}$ meaning that the family $\left\{f_{\varepsilon}\right\}$ describes all possible bifurcations occurring next to $f$. Indeed, to describe all possible bifurcations of a $k$-degenerate periodic orbit one needs at least $k+1$ parameters and $k$ can be arbitrary large.

Once Theorem 1 is proved the proof of Theorem 3 can be completed by inductive application of the following idea. Let $f$ be a $C^{s}$ diffeomorphism from a Newhouse domain $\mathcal{N} \subset \operatorname{Diff}^{r}(M)$ with a $k$-degenerate periodic orbit $p$ of period, say $n$, of $f$ for $s>k \geq r$, then $p$ is flat periodic point along the central manifold with respect to the $C^{r}$ topology, namely, by a $C^{r}$-perturbation one can make the restriction to the central manifold be the identical map. It allows us either to create a curve of periodic orbits or split p into any ahead given number of hyperbolic periodic orbits of the same period (or double the period of $p$ ) by a small perturbation. Since, created periodic orbits are hyperbolic they persist under perturbations. Moreover, after a perturbation we are still in a Newhouse domain one can iterate this procedure of creating a $k$-degenerate periodic orbits and splitting them without destroying what was done in previous stages (see section 2.6).

In what follows we need a few notions related to a saddle periodic point. These definitions will be needed in the proof of Theorem 4 .

Definition 3. Let $f$ be a $C^{s}$ diffeomorphism of a 2-dimensional manifold $M$ and let $p$ be a saddle periodic point of period $m$, namely, $f^{m}(p)=p$ with eigenvalues $\lambda$ and $\mu, \lambda<1<\mu$. The saddle exponent of $p$ is the number $\rho(p, f)=\frac{-\log \lambda}{\log \mu}$. We call $p$ a $\rho$-shrinking saddle, where $\rho=\rho(p, f)$. If $\rho$ is greater than some $r$, then $p$ is also called at least $r$-shrinking.

A saddle $p$ is called nonresonant if for any pair of positive integers $n$ and $m$ such that the number $\lambda^{n} \mu^{m}$ is different from 1 .

2.1. A Scheme of a Proof of Theorem 4. Theorem 4 is stated in ([GST], Thm.4). A proof of this theorem is outlined there. Proof of several technical statements 1 is omitted there. We present a rigorous proof which essentially uses ideas given in [GST]. In what follows a $C^{r}$-perturbation means a small $C^{r}$-perturbation. The proof of Theorem 1 consists of four steps.

The first step. From the existence of a homoclinic tangency of a dissipative saddle, we deduce the existence (after a $C^{r}$-perturbation) of a homoclinic tangency of an at least $k$ shrinking saddle, $k>r$.

The second step. From the existence of a homoclinic tangency of an at least $k$-shrinking saddle, we create a $k$-floor tower (defined in section 2.4) after a $C^{r}$-perturbation (see Fig.3 for $k=3$ ).

The third step. From the existence of a $k$-floor tower, we show that a $C^{r}$-perturbation can make a $k$-th order homoclinic tangency.

\footnotetext{
${ }^{1}$ Lemmas 1 and 2 in GST which corresponds to Lemmas 1 and 3 of the present paper respectively
} 
The fourth step. From the existence of a $k$-th order homoclinic tangency we construct by a $C^{r}$-perturbation a $k$-th order degenerate periodic orbit of an arbitrarily high period.

Notice that the way we construct a $k$-tower is slightly different from the one in [GST.

The proof of Theorem 1 is given in sections 2.2-2.5 according to the following plan. In section 2.2 we present some basic properties of a return map in a neighborhood of a quadratic homoclinic tangency. In section 2.3 we realize the first step (Corollary 1 ) and calculate limits for return maps in a neighborhood of a $k$-th order homoclinic tangency, where $k \geq 2$. The second and the third steps are done in sections 2.4 and 2.5 respectively. The last fourth step consists in application of Corollary 3 proven in section 2.3.

\subsection{Basic properties of a return map in a neighborhood of a homoclinic tangency.}

Fix a positive integer $r \geq 2$. Consider a $C^{\infty}$ smooth diffeomorphism $f: M^{2} \rightarrow M^{2}$ with a saddle fixed point $p$, namely, $f(p)=p$ with the eigenvalues $\lambda$ and $\mu$. Assume the saddle $p$ is dissipative and nonresonant. We can obtain all conditions by applying a $C^{r}$-perturbation (for $f \in C^{r}$, or/and $\lambda \mu=1$, or/and by inverting $f$ ) if necessary. Then by the standard fact from the theory of normal forms e.g. [IY] the map $f$ is $C^{r}$ linearizable in a neighborhood $U$ of $p$

$$
f:(x, y) \mapsto(\lambda x, \mu y),
$$

where $\lambda<1<\mu$ and $\lambda \mu<1$. The larger is $r$, the smaller is the neighborhood $U$, where a $C^{r}$-normal form applicable.

Assume that the stable $W^{s}(p)$ and unstable manifold $W^{u}(p)$ of $p$ in normal coordinates have a point of quadratic tangency $q$ with coordinates $(1,0)$ and for some $N$ we have that $f^{-N}(q)=\tilde{q}$ has coordinates $(0,1)$ (see Fig. 1). Assume also that in a neighborhood of the homoclinic point $q$ the unstable manifold $W^{u}(p)$ lies in the upper half plane $\{y \geq 0\}$ and the directions of $W^{u}(p)$ and $W^{s}(p)$ at the point of tangency $q$ are the same (see Fig.1). Diffeomorphisms with such type of homoclinic tangency are dense in a Newhouse domain see e.g. [PT].

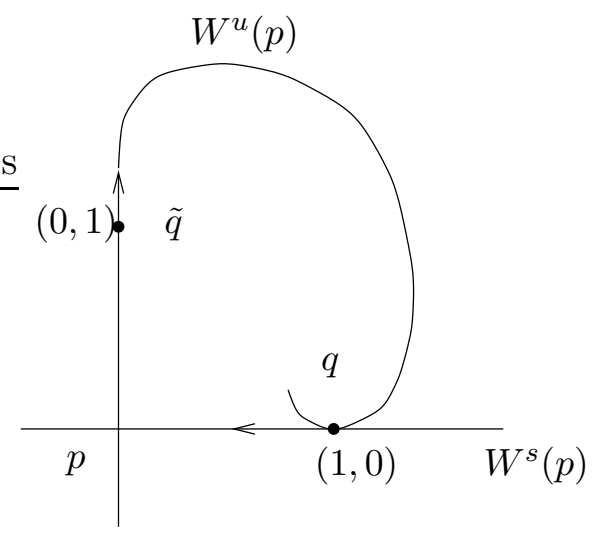

FiguRE 1. Homoclinic tangency

Rescale sufficiently small neighborhoods $U$ and $\tilde{U}$ of $q$ and $\tilde{q}$ respectively. Denote by $W_{l o c}^{u}(p)$ the first connected component of the intersection $W^{u}(p) \cap U$ (see Fig. 2). Below we shall use the coordinate systems in $U$ and $\tilde{U}$ induced by the normal coordinates of $p$ and $f$. Write $W_{l o c}^{u}(p)$ in $U$ as the graph of a function $y=c x^{2}+g(x)$, where $g(x)=o\left(x^{2}\right), c>0$. A 
rectangle in $U$ (resp. $\tilde{U}$ ) is called a right rectangle if it has two side that are parallel to the coordinate axis.

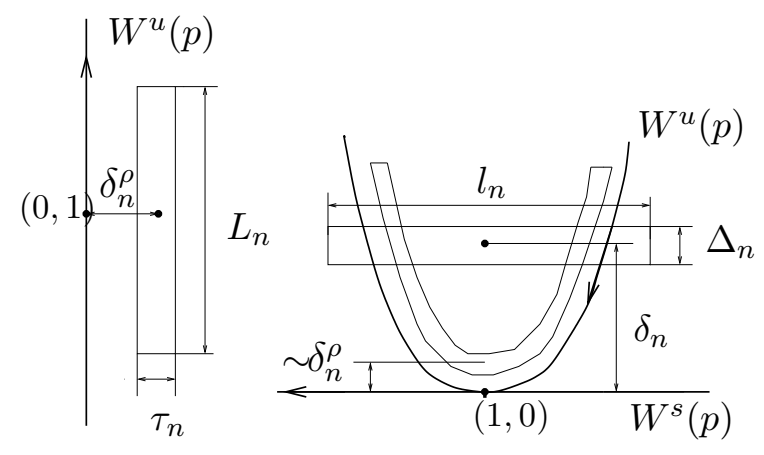

Figure 2. Neighborhoods of points of homoclinic tangencies.

Proposition 1. Let $c>0$ be the above constant and $n \in \mathbb{Z}_{+}$be sufficiently. Put $\delta_{n}=$ $\mu^{-n}, \Delta_{n}=2 \delta_{n}^{3 / 2}$, and $l_{n}=3 c \delta_{n}^{1 / 2}$. Consider a right rectangle $T_{n}$ centered at $\left(1, \delta_{n}\right)$ whose horizontal (resp. vertical) length is $l_{n}$ (resp. $\Delta_{n}$ ). Then the image $f^{n}\left(T_{n}\right)$ is the right rectangle centered at $\left(\delta_{n}^{\rho}, 1\right)$ whose horizontal (resp. vertical) length is $\tau_{n}=3 c \delta_{n}^{1 / 2} \lambda^{n}$ (resp. $\left.L_{n}=2 \delta_{n}^{1 / 2}\right)$. Moreover, $T_{n}$ and $f^{n+N}\left(T_{n}\right)$ form a horseshoe which has a periodic saddle $p^{\prime}$ of period $n+N$ and the curvilinear rectangle $f^{n+N}\left(T_{n}\right)$ is $C \delta_{n}^{\rho}$ distant away from $W^{s}(p)$ for some $C>0$ independent of $n$ (see Fig. 2).

Remark 1. The exponent $\rho=\frac{\log 1 / \lambda}{\log \mu}$ gives a characteristic of distortion while a trajectory pass in a neighborhood of saddle $p$ in the following sense. The rectangle $T_{n}$ is $\delta_{n}$-distance away from $W^{s}(p)$, but its image $f^{n+N}\left(T_{n}\right)$ is $\delta_{n}^{\rho}$-distance away from $W^{s}(p)$, so the more exponent $\rho=\frac{\log 1 / \lambda}{\log \mu}$ of saddle $p$, the deeper the horseshoe part $f^{n+N}\left(T_{n}\right)$ goes inside $W_{\text {loc }}^{u}(p)$ with respect to $T_{n}$ and gets closer to $W^{s}(p)$.

Proof of Proposition 10: Use the fact that $f$ is linear (4) in the unit square around $p$ to prove the first part. Since $N$ is fixed the ratio of $\left\{\right.$ distance of the rectangle $f^{n}\left(T_{n}\right)$ to $\left.W^{u}(x)\right\}$ and $\left\{\right.$ distance of the curvilinear rectangle $f^{n+N}\left(T_{n}\right)$ to $\left.W_{l o c}^{u}(x)\right\}$ is bounded. This implies the second statement of the Proposition and completes the proof. Q.E.D.

2.3. The first step: higher order homoclinic tangencies and appropriate limits. It is well-known from e.g. $\mathbb{M V}], \mathbb{P T}]$, and $\left[\mathrm{TY} \|\right.$ that for a generic 1-parameter family $\left\{f_{\epsilon}\right\}$ unfolding a quadratic (1-st order) homoclinic tangency $q$ and for any number $c \in[-2,1 / 4]$ there exist three sequences $\left(n \in \mathbb{Z}_{+}\right)$: rectangles $T_{n}$ next to $q$, rescalings $R_{n}: T_{n} \rightarrow[-2,2] \times$ $[-2,2]$, and parameters $\epsilon_{n}$ such that a return map $f_{\epsilon_{n}}^{n}$ from $T_{n}$ into itself converges to the map $(x, y) \rightarrow\left(y, y^{2}+c\right)$. $T_{n}$ and $R_{n}$ are independent of $c$, but $\epsilon_{n}=\epsilon_{n}(c)$ depends on $c$.

In this section we show that for a generic $k$-parameter family $\left\{f_{\mu}\right\}$ unfolding a $(k-1)$-st order homoclinic tangency $q$ and for any set of numbers $M=\left(M_{0}, \ldots, M_{k-1}\right) \in \mathbb{R}^{k}$ there exist three sequences $\left(n \in \mathbb{Z}_{+}\right)$: rectangles $T_{n}$ next to $q$, rescalings $R_{n}: T_{n} \rightarrow[-2,2] \times[-2,2]$, and parameters $\mu(n)=\left(\mu_{0}(n), \ldots, \mu_{k-1}(n)\right)$ such that a return map $f_{\mu(n)}^{n}$ from $T_{n}$ into itself under $f_{\epsilon_{n}}$ converges to $(x, y) \rightarrow\left(y, y^{k}+\sum_{i=0}^{k-1} M_{i} y^{i}\right)$. Moreover, convergence holds with 
respect to the $C^{r}$ topology for any $r \in \mathbb{Z}_{+}$. The calculation presented below is in the spirit of [PT] and [TY].

Consider a $C^{\infty}$ diffeomorphism $f$ which has a dissipative saddle periodic point $p$ exhibiting a homoclinic tangency of $(k-1)$-st order. Figure 1 illustrates the topology for even $k$. We shall use notations of Fig. 1. Take coordinates $(\bar{x}, \bar{y})=(x-1, y)$ around the homoclinic point $q$ and coordinates $(\tilde{x}, \tilde{y})=(x, y-1)$ around the homoclinic point $\tilde{q}$.

Because of a $(k-1)$-st order tangency, the map $f^{N}:(\tilde{x}, \tilde{y}) \rightarrow(\bar{x}, \bar{y})$ from a neighborhood $\tilde{U}$ of $\tilde{q}$ with coordinates $(\tilde{x}, \tilde{y})$ to a neighborhood $U$ of $q$ with coordinates $(\bar{x}, \bar{y})$ can be written in the form:

$$
\left(\begin{array}{c}
\tilde{x} \\
\tilde{y}
\end{array}\right) \stackrel{f^{N}}{\longmapsto}\left(\begin{array}{c}
\alpha \tilde{y}+\beta \tilde{x}+H_{1}(\tilde{x}, \tilde{y}) \\
\gamma \tilde{y}^{k}+\sigma \tilde{x}+H_{2}(\tilde{x}, \tilde{y})
\end{array}\right),
$$

where $\alpha, \beta$, and $\gamma$ are constants such that for $\tilde{x}=\tilde{y}=0$

$$
\left\{\begin{array}{l}
H_{1}=\partial_{x} H_{1}=\partial_{y} H_{1}=0 \\
H_{2}=\partial_{x} H_{2}=\partial_{y}^{j} H_{2}=0, \quad j=1, \ldots, k .
\end{array}\right.
$$

To see that formula (5) holds consider images of lines $\{\tilde{x}=$ constant $\}$.

Consider a generic $k$-parameter unfolding of a $(k-1)$-st order homoclinic tangency:

$$
\left(\begin{array}{c}
\tilde{x} \\
\tilde{y}
\end{array}\right) \stackrel{f_{\mu}^{N}}{\longmapsto}\left(\begin{array}{c}
\alpha \tilde{y}+\beta \tilde{x}+H_{1}(\tilde{x}, \tilde{y}) \\
\gamma \tilde{y}^{k}+\sum_{i=0}^{k-1} \mu_{i} \tilde{y}^{i}+\sigma \tilde{x}+H_{2}(\tilde{x}, \tilde{y})
\end{array}\right) .
$$

The main result of this section in the following:

Lemma 1. With the above notations and $k \geq 2$ for an arbitrary set of real numbers $\left\{M_{i}\right\}_{i=0}^{k-1}$ there exists a sequence of parameters $\{\mu(n)\}_{n \in \mathbb{Z}_{+}}$such that $\mu(n)$ tends 0 as $n \rightarrow \infty$ U and a sequence of change of variables $R_{n}:(\bar{x}, \bar{y}) \rightarrow\left(x_{n}, y_{n}\right)$ such that the sequence of maps: $\left\{R_{n} \circ f_{\mu(n)}^{n+N} \circ R_{n}^{-1}:[-2,2] \times[-2,2] \rightarrow[-2,2] \times[-2,2]\right\}$ converges to the 1-dimensional map

$$
\left(\begin{array}{l}
x \\
y
\end{array}\right) \stackrel{\phi_{M}}{\longmapsto}\left(\begin{array}{c}
y \\
y^{k}+\sum_{i=0}^{k-1} M_{i} y^{i}
\end{array}\right)
$$

in the $C^{r}$ topology for any $r$.

Corollary 3. (The fourth step of the proof of Theorem 4 ) For $M_{1}=1$, and $M_{j}=0, j=$ $0,2, \ldots, k-1$ by a $C^{r}$-perturbation of a $C^{\infty}$ diffeomorphism $f$ exhibiting a homoclinic tangency of order $(k-1)$ one can create a $C^{r}$ diffeomorphism $\tilde{f}$ with a $(k-1)$-degenerate periodic orbit of an arbitrarily high period.

Corollary 4. For $k=2, M_{0}=-2$, and $M_{1}=0$ by a $C^{r}$-perturbation of a $C^{\infty}$ diffeomorphism $f$ exhibiting a quadratic homoclinic tangency one can create a $C^{\infty}$ diffeomorphism $f$ with a periodic saddle $p$ exhibiting a homoclinic tangency and eigenvalues of $p$ are close to 2 and to +0 respectively.

\footnotetext{
${ }^{2}$ see (11) for the exact form of the sequence
} 
To prove this corollary recall that for any $r$ the map $(x, y) \rightarrow\left(y, y^{2}-2\right), x, y \in[-2,2]$ has a fixed point $(2,2)$. One can show that by a $C^{r}$ perturbation of this 2-dimensional map a fixed point $(2,2)$ becomes a saddle near to $(2,2)$ exhibiting a homoclinic tangency. In $\|\mathrm{PT}\|$ $\S 6.3$ Prop.3 figures 6.4 and 6.5 or $\mathbb{M V}$ pg.14, this is shown to be true. On 2-dimensional perturbations of the 1 -dimensional map $y \mapsto y^{2}-\mu$ see also $[\mathrm{BQ}]$.

Proof of Lemma 1: We follow the standard method and split the return map $f^{n+N}$ into the composition of two maps: the linear map $f^{n}:(x, y) \rightarrow\left(\lambda^{n} x, \mu^{n} y\right)$ and the map $f_{\mu}^{N}$ given by formula (7). The composition of $f_{\mu}^{N}$ and $f^{n}$ has the form:

$$
\left(\begin{array}{c}
\bar{x} \\
\bar{y}
\end{array}\right) \stackrel{f_{\mu}^{N} \circ f^{n}}{\longmapsto}\left(\begin{array}{c}
\alpha \bar{y}_{n}+\beta \lambda^{n}(1+\bar{x})+H_{1}(\cdot, \cdot) \\
\gamma \bar{y}_{n}^{k}+\sum_{i=0}^{k-1} \mu_{i} \bar{y}_{n}^{i}+\sigma \lambda^{n}(1+\bar{x})+H_{2}(\cdot, \cdot)
\end{array}\right)
$$

where $\bar{y}_{n}=\mu^{n} \bar{y}-1, H_{j}(\cdot, \cdot)=H_{j}\left(\lambda^{n}(1+\bar{x}), \bar{y}_{n}\right), j=1,2$. Denote $\mu^{1 /(k-1)}$ by $\tau$. Introduce the change of variables $R_{n}:(\bar{x}, \bar{y}) \rightarrow\left(x_{n}, y_{n}\right)$, where

$$
\left(\begin{array}{l}
x_{n} \\
y_{n}
\end{array}\right)=\left(\begin{array}{c}
\tau^{n} \bar{x} \\
\tau^{n}\left(\mu^{n} \bar{y}-1\right)
\end{array}\right)
$$

In $\left(x_{n}, y_{n}\right)$-coordinates the map $f_{\mu}^{N} \circ f^{n}$ has the form:

$$
\left(\begin{array}{l}
x_{n} \\
y_{n}
\end{array}\right) \stackrel{f_{\mu}^{N} \circ f^{n}}{\longmapsto}\left(\begin{array}{c}
\alpha y_{n}+\beta \lambda^{n}\left(\tau^{n}+x_{n}\right)+\tau^{n} H_{1}(\cdot, \cdot) \\
\gamma y_{n}^{k}+\sum_{i=0}^{k-1} \mu_{i} \tau^{n(k-i)} y_{n}^{i}+\sigma \lambda^{n} \mu^{n}\left(\tau^{n}+x_{n}\right)+\tau^{k n} H_{2}(\cdot, \cdot)-\tau^{n}
\end{array}\right),
$$

where $H_{j}(\cdot, \cdot)=H_{j}\left(\lambda^{n}\left(1+\tau^{-n} x_{n}\right), \tau^{-n} y_{n}\right)$ for $j=1,2$.

Recall that $p$ is dissipative, so $\lambda \mu<1$ and $\lambda \tau<1$ too. Thus, condition (6) and $0<$ $\lambda, \tau^{-1}<1$ imply that terms $\lambda^{n} \mu^{n} x_{n}, \beta \lambda^{n}\left(\tau^{n}+x_{n}\right), \tau^{n} H_{1}\left(\lambda^{n}\left(1+\tau^{-n} x_{n}\right), \tau^{-n} y_{n}\right)$, and $\tau^{k n} H_{2}\left(\lambda^{n}\left(1+\tau^{-n} x_{n}\right), \tau^{-n} y_{n}\right)$ tends to 0 as $n \rightarrow \infty$ in the $C^{r}$ topology for any positive integer $r$.

Put

$$
\begin{array}{r}
\mu_{0}(n)=\mu^{-k n /(k-1)} M_{0}-\sigma \lambda^{n}+\mu^{-n} \\
\mu_{i}(n)=\mu^{-(k-i) n /(k-1)} M_{i} \text { for } i=1, \ldots, k-1 .
\end{array}
$$

We see that all $\left\{\mu_{i}(n)\right\}$ tends to 0 as $n$ tends to infinity. Therefore, in the limit as $n \rightarrow \infty$ we obtain

$$
\left(\begin{array}{c}
\tilde{x} \\
\tilde{y}
\end{array}\right) \stackrel{\phi_{M}}{\longmapsto}\left(\begin{array}{c}
\alpha y \\
\gamma y_{n}^{k}+\sum_{i=0}^{k-1} M_{i} y^{i}
\end{array}\right) .
$$

Additional change of variables depending on $\alpha$ and $\gamma$ completes the proof. Q.E.D.

2.4. The second step: Construction of a $k$-floor tower. Consider a $C^{\infty}$ diffeomorphism $f$ with a nonresonant saddle periodic point $p$ exhibiting a homoclinic tangency at a point $q$. First, we give a definition of $a k$-floor tower. Recall that $U$ denotes a neighborhood of the homoclinic tangency $q$. Let $\tilde{p}$ be a saddle periodic orbit of $f, \tilde{p} \in U$. Then denote by $W_{\text {loc }}^{s}(\tilde{p})$ (resp. $\left.W_{\text {loc }}^{u}(\tilde{p})\right)$ the first connected component of the intersection of stable (resp. unstable) manifold $W^{s}(\tilde{p})$ (resp. $\left.W^{u}(\tilde{p})\right)$ with $U$.

Definition 4. A $k$-floor tower is a contour consisting of $k$ saddle periodic points $p_{1}, \ldots, p_{r}$ (of different periods) such that $W_{\text {loc }}^{u}\left(p_{i}\right)$ is tangent to $W_{l o c}^{s}\left(p_{i+1}\right)$ for $i=1, \ldots, k-1$, and $W_{\text {loc }}^{u}\left(p_{k}\right)$ intersects $W_{\text {loc }}^{\text {s }}\left(p_{1}\right)$ transversally (see Fig.3 for $k=3$ ). 
Construction of a $k$-floor tower is an intermediate step in the proof of Theorem 4 . In this section we prove that

Lemma 2. For any positive integer $k$ a $C^{r}$ diffeomorphism $f$ exhibiting a homoclinic tangency for an at least $r$-shrinking saddle periodic orbit $p$ admits a $C^{r}$-perturbation $\tilde{f}$ such that $\tilde{f}$ has a $k$-floor tower. If $q$ is a point of homoclinic tangency of $f$, then the aforementioned tower of $\tilde{f}$ is located in a neighborhood $U$ of $q$.

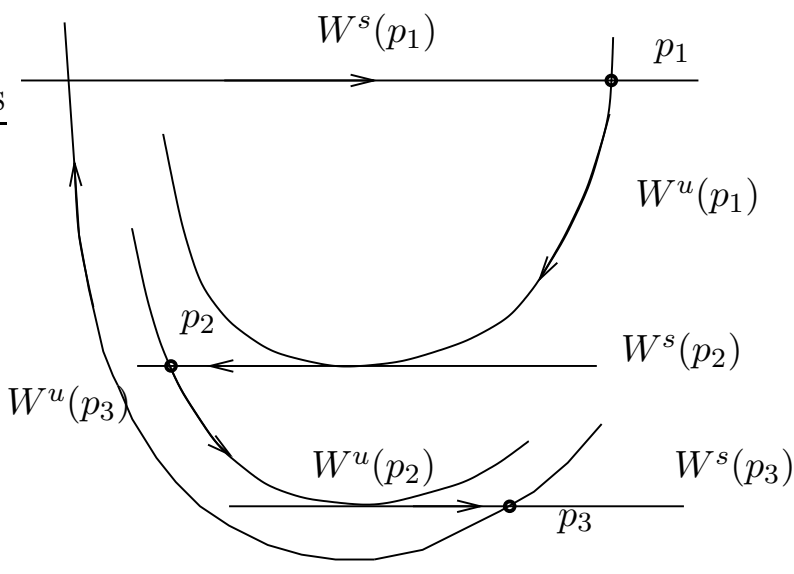

Figure 3. A 3-floor tower.

Proof: We prove this Lemma using localized perturbation technic. As usual consider normal coordinates for a nonresonant saddle $p$. Induce coordinates in $U$ by normal coordinates for the point $p$ and the diffeomorphism $f$. Application of Proposition 1 gives existence of the contour described on Fig. 4 in the case $k=3$. Indeed, consider an increasing sequence of numbers $n_{1}, \ldots, n_{k}$ such that for each $i=1, \ldots, k$ the following two properties hold:

1) $T_{n_{i}}$ intersects $f^{n_{i}+N}\left(T_{n_{i}}\right)$ and they form a horseshoe;

2) $n_{i+1}$ is the largest number such that $T_{n_{i+1}}$ and $f^{n_{i}+N}\left(T_{n_{i}}\right)$ intersect in a horseshoe-like way, i.e., that they bound an open set.

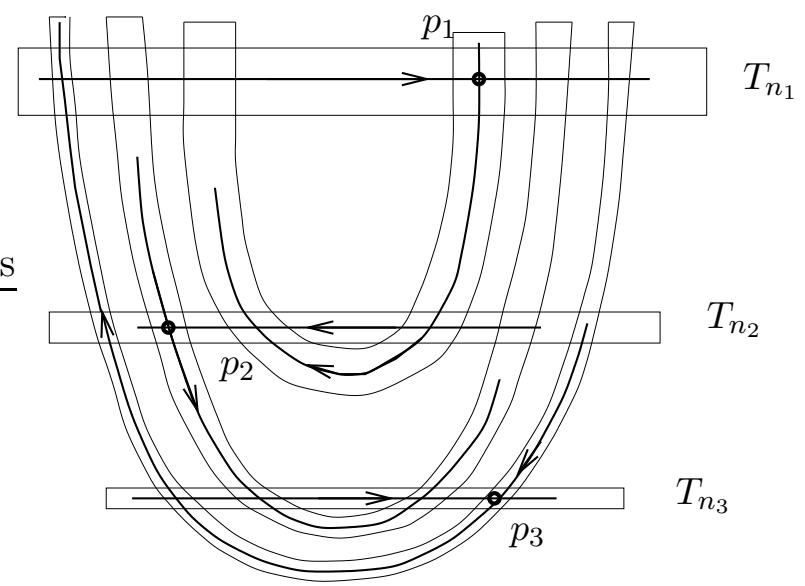

Figure 4. An uncomplete 3-floor tower. 
For each $i=1, \ldots, k$ condition 1 ) implies existence of a saddle periodic point $p_{i} \in T_{n_{i}} \cap$ $f^{n_{i}+N}\left(T_{n_{i}}\right)$ of period $n_{i}+N$ and condition 2) that $W_{l o c}^{s}\left(p_{i+1}\right)$ and $W_{l o c}^{u}\left(p_{i}\right)$ intersect.

Let $U$ be equipped with normal coordinates. Define the maximal distance in the vertical direction between $W_{l o c}^{s}\left(p_{i}\right)$ and $W_{l o c}^{u}\left(p_{i}\right)$ as the maximum of distance between any two points $x \in W_{l o c}^{s}\left(p_{i}\right)$ and $y \in W_{l o c}^{u}\left(p_{i}\right)$ such that $x$ and $y$ have the same $\bar{x}$-coordinate. Denote the vertical distance between centers of $T_{n_{i}}$ and $T_{n_{i+1}}$ by $t_{i}$ (see Fig. 5). By calculation in section 2.2 we get $t_{i}=\mu^{-n_{i}}-\mu^{-n_{i+1}}$.

Proposition 2. If the saddle p having a homoclinic tangency is at least $r$-shrinking, then the ratio $\frac{s_{i}-t_{i}}{t_{i}^{r}}$ is arbitrarily small for each $i=1, \ldots, k-1$.

Proof: Let us use notations and quantitative estimate obtained in Proposition 1. Let $p$ be $\rho$-shrinking, $\rho>r$. Recall that the rectangle $T_{n}$ is centered at $\left(1, \delta_{n}=\mu^{-n}\right)$ and has length $3 c \mu^{-n / 2}$ and width $\mu^{-3 n / 2}$. Notice that the width is much less than $\mu^{-n}$, the height of center $\mu^{-n}$. Since $p$ is $\rho$-shrinking and $n_{i}$ and $n_{i+1}$ satisfy the conditions $\mu^{-n_{i+1}}>$ const $\mu^{\rho n_{i}}>$ $\mu^{-n_{i+1}-1}$ it implies that $s_{i}-t_{i}<\delta_{n_{i+1}}+\Delta_{n_{i}}<C \delta_{n_{i}}^{\rho}<\epsilon \delta_{n_{i}}^{r}=\epsilon t_{n_{i}}$ for any $\epsilon>0$ and a sufficiently large $n_{i}$ (see Fig.2 right and Fig.5). Q.E.D.

Proposition 3. If the ratio $\left(s_{i}-t_{i}\right) / t_{i}^{r}$ is arbitrarily small, then there exists a small $C^{r}$ perturbation inside of the ball $B$ (see Fig.5) such that $W_{l o c}^{s}\left(p_{n_{i+1}}\right)$ and $W_{\text {loc }}^{u}\left(p_{n_{i}}\right)$ have a point of a heteroclinic tangency.

Proof: Using the standard perturbation technic we lift up $W_{l o c}^{u}\left(p_{n_{i}}\right)$ and create a heteroclinic tangency. Q.E.D.

Remark 2. In order to construct a $k$-floor tower one needs to create a heteroclinic tangency of $W_{l o c}^{s}\left(p_{n_{i+1}}\right)$ and $W_{l o c}^{u}\left(p_{n_{i}}\right)$ by a $C^{r}$-perturbation. We construct it by "bending" $W_{l o c}^{u}\left(p_{n_{i}}\right)$.

Another way to construct it is by fixing the eigenvalue $\mu>1$ and varying the other eigenvalue $\lambda<1$ of the saddle p exhibiting homoclinic tangency. See Proposition 1 : the rectangle $T_{n}$ is centered at $\left(1, \mu^{-1}\right)$ and the curvilinear rectangle $f^{n+N}\left(T_{n}\right)$ is $C \delta_{n}^{\rho}=C \lambda^{n}$ distant away from $W^{s}(p)$, therefore, by changing $\lambda$ one can vary the position of $f^{n+N}\left(T_{n}\right)$ without changing the position of $T_{n}$. But, in this case one needs some additional geometric argument to construct all heteroclinic tangencies of a $k$-tower simultaneously.

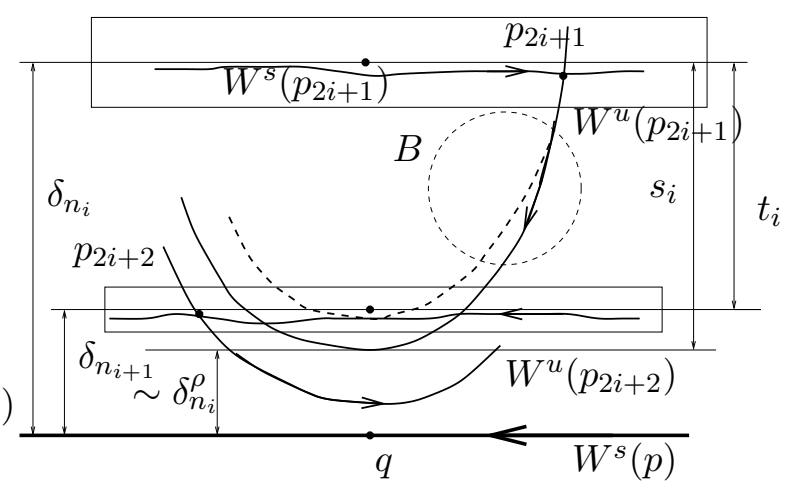

FiguRE 5. A localized perturbation for a floor of a tower.

Proposition 4. Given $r \in \mathbb{Z}_{+}$if $n_{1}=n_{1}(r)$ is sufficiently large, then all saddles $p_{1}, \ldots, p_{k}$, described above, are at least $r$-shrinking. 
Proof: Recall that $p_{1}, \ldots, p_{k}$ are saddle periodic points located in a neighborhood of a homoclinic tangency of a dissipative saddle $p$. Let $\lambda<1<\mu$ denote eigenvalues of $p$.

With the notations of Lemma 1 the return map $f^{n+N}$ defined on the rectangle $T_{n}$ (see section 2.2) has the form

$$
\left(\begin{array}{c}
\bar{x} \\
\bar{y}
\end{array}\right) \mapsto\left(\begin{array}{c}
\alpha \bar{y}_{n}+\beta \lambda^{n}(1+\bar{x})+H_{1}(\cdot, \cdot) \\
\gamma y_{n}^{2}+\sigma \lambda^{n}(1+\bar{x})+H_{2}(\cdot, \cdot)
\end{array}\right),
$$

where $\bar{y}_{n}=\mu^{n} \bar{y}-1, H_{j}(\cdot, \cdot)=H_{j}\left(\lambda^{n}(1+\bar{x}), \bar{y}_{n}\right), j=1,2$. The functions $H_{1}$ and $H_{2}$ satisfy condition (6). Compare with (9) for $k=2, \mu_{i}=0, i=0,1$.

Therefore, in our notations the fixed point equation has the form

$$
\left\{\begin{array}{l}
\alpha \bar{y}_{n}+\beta \lambda^{n}(1+\bar{x})+H_{1}\left(\lambda^{n}(1+\bar{x}), \bar{y}^{n}\right)=\bar{x}, \\
\gamma \bar{y}_{n}^{2}+\sigma \lambda^{n}(1+\bar{x})+H_{2}\left(\lambda^{n}(1+\bar{x}), \bar{y}_{n}\right)=\bar{y}
\end{array}\right.
$$

Since, the saddle $p$ is dissipative all terms $\beta \lambda^{n}(1+\bar{x}), H_{1}\left(\lambda^{n}(1+\bar{x}), \bar{y}^{n}\right), \sigma \lambda^{n}(1+\bar{x})$, and $H_{2}\left(\lambda^{n}(1+\bar{x}), \bar{y}_{n}\right)$ tend to zero as $n$ tends to $+\infty$. Denote one of fixed points by $\left(\bar{x}_{n}^{0}, \bar{y}_{n}^{0}\right)$. It should belong to $T_{n}$ which implies that $\bar{y}_{n}^{0}=\mu^{-n}(1+o(1))$. Direct calculation of the linear part at $\left(\bar{x}_{n}^{0}, \bar{y}_{n}^{0}\right)$ shows that eigenvalues of the linearization approach $2 \mu^{n}$ and $\lambda^{n} / 2$ respectively. Therefore, if $n$ is sufficiently large, then $p_{n}$ is obviously $r$-shrinking for any ahead given $r$. This completes the proof. Q.E.D.

2.5. The third step: Construction of a $k-t h$ order tangency. We shall prove that by a perturbation of a $(k+1)$-floor tower one can create a $k$-th order homoclinic tangency. Let us start with a 2-nd order tangency and use induction in $k$ then.

Proposition 5. GST A perturbation of a 3-floor tower can create a 2-nd order homoclinic tangency.

Proof: Step 1. Let us create a 1-st order tangency of $W_{l o c}^{u}\left(p_{2}\right)$ and $W_{l o c}^{s}\left(p_{1}\right)$. Start with a 3-tower on Fig.3. "Push" $W_{l o c}^{u}\left(p_{2}\right)$ down $W_{l o c}^{s}\left(p_{3}\right)$. Denote by $\gamma$ the tongue (the part) of $W_{l o c}^{u}\left(p_{2}\right)$ underneath $W_{l o c}^{s}\left(p_{3}\right)$. The tongue $\gamma$ is in the sector of the saddle hyperbolic point $p_{3}$, therefore, under iteration of $f \gamma$ will be stretched along $W_{l o c}^{u}\left(p_{3}\right)$ and for some $s$ $f^{s}(\gamma) \cap W^{s}\left(p_{1}\right) \neq \emptyset$. Varying the size of the tongue $\gamma$ we can create a heteroclinic tangency (see Fig.6.a) with $i=0$ ). Denote a point of tangency by $q^{*}$. Only two parts of $W^{u}\left(p_{2}\right)$ are depicted on figure 6 a): first part — starting part of $W^{u}\left(p_{2}\right)$ at $p_{2}$ and second - image of $\gamma$ after a number of iterations under $f$ (in above notations $f^{s}(\gamma)$ ).

Assume that saddle $p_{1}$ is nonresonant. Then there is normal coordinates around $p_{1}$ linearizing $f$. Induce by $f$ normal coordinates in a neighborhood of $U^{*}$ of $q^{*}$. In what follows we shall use these coordinate systems in $U^{*}$.

Step 2. Let us create a 2-nd order homoclinic tangency of $W_{l o c}^{u}\left(p_{1}\right)$ and $W_{l o c}^{s}\left(p_{1}\right)$. Start with a contour on Fig.6 a). "Push" $W_{l o c}^{u}\left(p_{1}\right)$ down $W_{l o c}^{s}\left(p_{2}\right)$. Denote by $\gamma^{1}$ the tongue (the part) of $W^{u}\left(p_{1}\right)$ underneath $W^{s}\left(p_{2}\right)$. Some iterate of the tongue $\gamma^{1}$ of $W_{l o c}^{u}\left(p_{1}\right)$ come into $U^{*}$. $U^{*}$ has normal coordinates and naturally defined the horizontal and the vertical directions. Now our goal is varying the size of $\gamma^{1}$ construct a tangency of some iterate of $\gamma^{1}$ to the horizontal direction in $U^{*}$. 


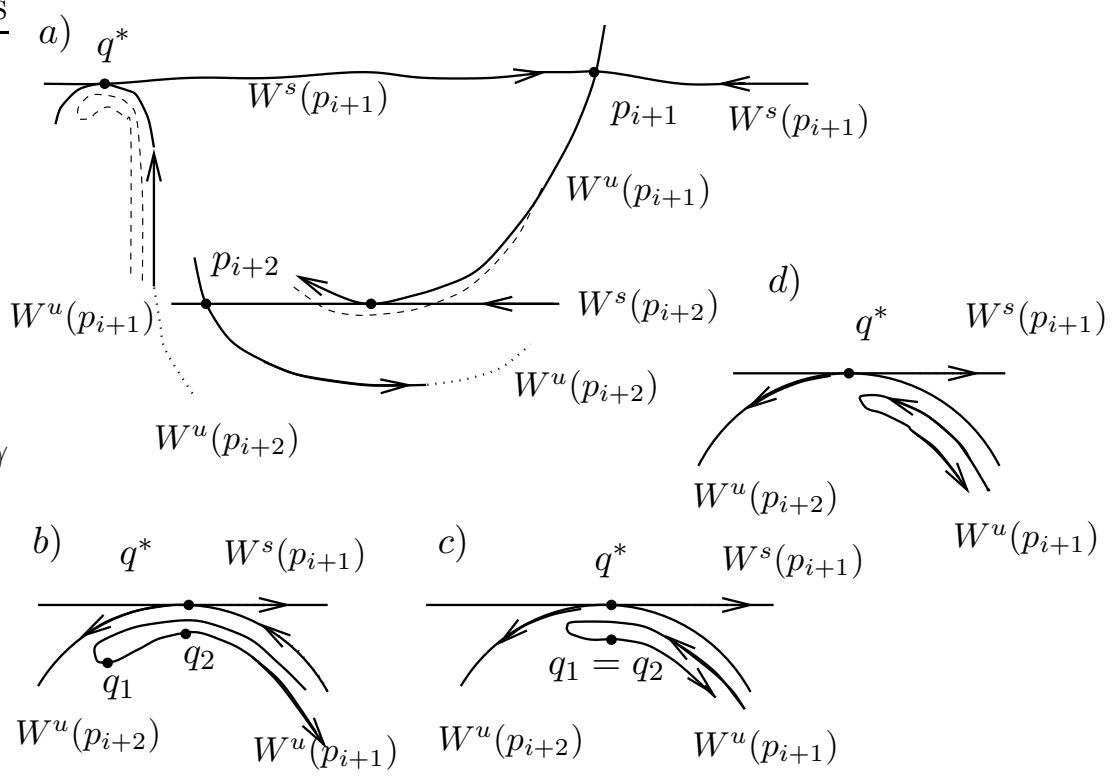

FiguRE 6. a 2-nd (even) order tangency.

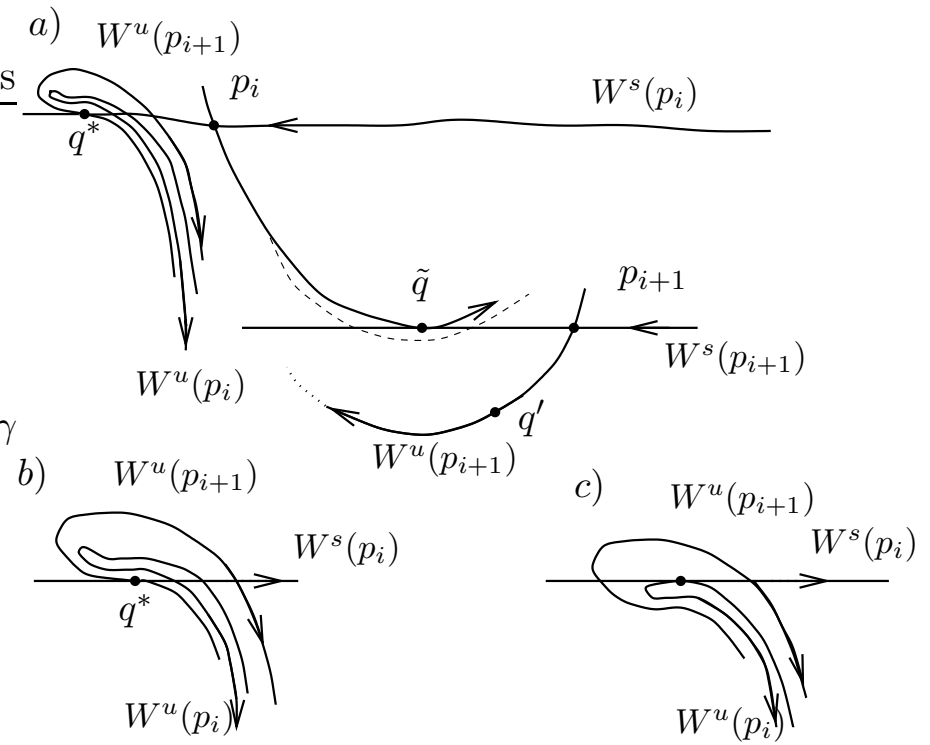

FiguRE 7. an odd order tangency.

Fix some coordinates in a neighborhood of $\tilde{U}$ of a tangency $\tilde{q}$ of $W_{l o c}^{u}\left(p_{1}\right)$ and $W_{l o c}^{s}\left(p_{2}\right)$. Consider a 1-parameter family of diffeomorphisms $\left\{f_{\epsilon}\right\}$, where $\epsilon$ is the maximal distance of $W_{l o c}^{u}\left(p_{1}\right) \cap \tilde{U}$ and $W_{l o c}^{s}\left(p_{2}\right) \cap \tilde{U}$ in the vertical direction.

Let $\gamma_{\epsilon}=W_{l o c}^{u}\left(p_{1}\right) \cap\{y \leq 0\}$. Fix $\epsilon>0$ and $s=s(\epsilon)$ such that $\gamma_{\epsilon}^{s}=f^{s}\left(\gamma_{\epsilon}^{1}\right) \cap U^{*} \neq \emptyset$ (see Fig. $6 \mathrm{~b})$ ). The curve $\gamma_{\epsilon}^{s}$ has two points $q_{1}$ and $q_{2}$ of tangency to the horizontal direction. As $\epsilon$ decreases $q_{1}$ and $q_{2}$ approach one to the other and for some critical value $\epsilon^{*}$ they collide and $q_{1}=q_{2}$ (see Fig. $6 \mathrm{c}$ )). 
At the point $q_{1}=q_{2} W_{l o c}^{u}\left(p_{1}\right)$ has a 2-nd order tangency to the horizontal direction. Let this point have coordinates $\left(\epsilon_{1}, \epsilon_{2}\right)$ in $U^{*}$. Lifting $W_{\text {loc }}^{u}\left(p_{2}\right)$ by $\epsilon_{2}$ we can create a 2 -nd order tangency at point $\left(\epsilon_{1}, 0\right)$. This completes the proof of the Proposition.

To construct a $k$-th order tangency assume that we have already constructed a diffeomorphism $f \in \operatorname{Diff}^{r}(M)$ which has a saddle periodic point $p$ exhibiting a homoclinic tangency. In a small neighborhood of homoclinic tangency there exist two saddle periodic orbits $p_{1}$ and $p_{2}$ such that $W_{l o c}^{s}\left(p_{1}\right)$ and $W^{u}\left(p_{2}\right)$ has a $(k-1)$-st order tangency and $W_{l o c}^{u}\left(p_{1}\right)$ and $W_{l o c}^{s}\left(p_{2}\right)$ have a tangency. Consider two case $k=2 p$ and $k=2 p+1$ (see Figures 6 a) and 7 a) for $i=0$ respectively). These cases have different topological pictures.

Lemma 3. Let $p_{1}$ and $p_{2}$ be two saddle periodic points and $W^{u}\left(p_{2}\right)$ have a $(k-1)$-st order tangency with $W_{\text {loc }}^{s}\left(p_{1}\right)$ at point $q^{*}$ and $W_{\text {loc }}^{u}\left(p_{1}\right)$ and $W_{\text {loc }}^{s}\left(p_{2}\right)$ have a tangency at a point $\tilde{q}$. Then by a $C^{r}$-perturbation one can create a $k$-th order homoclinic tangency of $W^{s}\left(p_{1}\right)$ and $W^{u}\left(p_{1}\right)$ (when $k$ is even see Fig. 6 a) for $i=0$ and when $k$ is odd see Fig.7 a) for $i=1$ ).

Proof: Assume that $p_{1}$ and $p_{2}$ are nonresonant saddles. Fix normal coordinate systems $\left(x_{1}, y_{1}\right)$ (resp. $\left(x_{2}, y_{2}\right)$ ) around $p_{1}$ (resp. $p_{2}$ ) so that $f$ is linear there. Let $\lambda<1<\mu$ be eigenvalues of $p_{2}$.

Denote by $q^{\prime}$ a preimage of $q^{*} q^{\prime}=f^{-N}\left(q^{*}\right)$. Fix the normal coordinate systems $(\hat{x}, \hat{y})$, $(\bar{x}, \bar{y})$, and $(\tilde{x}, \tilde{y})$ in neighborhoods $U^{*}$ of $q^{*}, \tilde{U}$ of $\tilde{q}$, and $U^{\prime}$ of $q^{\prime}$ respectively. In what follows we shall use the normal coordinate systems in $U^{*}$ and $\tilde{U}, U^{\prime}$ induced by $f$ from coordinate systems $\left(x_{1}, y_{1}\right)$ and $\left(x_{2}, y_{2}\right)$ respectively.

The tongue $W_{l o c}^{u}\left(p_{1}\right) \cap \tilde{U}$ has the form $\hat{y}=a \bar{x}^{2}+g(\bar{x})$, where $a>0$ and $g(\bar{x})=o\left(\bar{x}^{2}\right)$ at $\bar{x}=0$. The map $f^{N}: \tilde{U} \rightarrow U^{*}$ has the form

$$
\left(\begin{array}{c}
\tilde{x} \\
\tilde{y}
\end{array}\right) \mapsto\left(\begin{array}{c}
\alpha \tilde{y}_{n}+\beta \tilde{x}+H_{1}(\tilde{x}, \tilde{y}) \\
\gamma \tilde{y}^{k}+\sigma \tilde{x}+H_{2}(\tilde{x}, \tilde{y})
\end{array}\right)
$$

where $H_{1}(\tilde{x}, \tilde{y})$ and $H_{2}(\tilde{x}, \tilde{y})$ satisfy condition (6).

The idea of the proof is to shift $W_{l o c}^{u}\left(p_{1}\right)$ down to $\bar{y}=a \bar{x}^{2}-\epsilon+g(\bar{x})$ and consider the versal $k$ parameter family $\left\{f_{\mu}\right\}, \mu=\left(\mu_{0}, \ldots, \mu_{k-1}\right)$ of the form (17) unfolding $(k-1)$-st order tangency at the heteroclinic tangency at $q^{*}$. The part of $W_{l o c}^{u}\left(p_{1}\right)$ given by $\{\bar{y} \leq 0\} \cap\left\{\bar{y}=a \bar{x}^{2}-\epsilon+g(\bar{x})\right\}$ after a number of iterations under $f$ come to a neighborhood $U^{*}$ of $q^{*}$. It turns out that by varying $(k+1)$ parameters $\epsilon, \mu_{0}, \ldots, \mu_{k-1}$ we can construct a $k$-th order homoclinic tangency in $U^{*}$. Let us prove this statement.

Calculate the composition map $f_{\mu}^{N} \circ f^{n}: \tilde{U}_{n} \rightarrow U^{*}$, which is defined in an open subset $\tilde{U}_{n} \subset \tilde{U}$,

$$
\left(\begin{array}{c}
\hat{x} \\
\hat{y}
\end{array}\right) \stackrel{f_{\mu}^{N} \circ f^{n}}{\longmapsto}\left(\begin{array}{c}
\alpha \bar{y}_{n}+\beta \lambda^{n}(1+\bar{x})+H_{1}(\cdot, \cdot) \\
\gamma \bar{y}_{n}^{k}+\sum_{i=0}^{k-1} \mu_{i} \bar{y}_{n}^{i}+\sigma \lambda^{n}(1+\bar{x})+H_{2}(\cdot, \cdot)
\end{array}\right) .
$$

where $\bar{y}_{n}=\mu^{n}\left(a \bar{x}^{2}-\epsilon+g(\bar{x})\right)+1, H_{j}(\cdot, \cdot)=H_{j}\left(\lambda^{n}(1+\bar{x}), \bar{y}_{n}\right)$ and $j=1,2$. Assume that after a change of coordinates in $U^{*}$ preserving lines $\{\hat{y}=$ const $\}$ variable $\sigma$ has an appropriate sign depending on whether $k$ is odd or even and on high order derivatives of $y(x)=a \bar{x}^{2}+g(\bar{x})$.

Consider the following parameterization of $W_{l o c}^{u}\left(p_{1}\right) \cap\{\bar{y} \leq 0\}$ by $\bar{x}(t)=t, \bar{y}(t)=a t^{2}-$ $\epsilon+g(t)$. Let $\hat{x}_{n}(t)$ denote the first coordinate function of (16) and $\hat{y}_{n}(t)$ - the second. It is enough to find parameter values $\epsilon(n), \mu(n)=\left(\mu_{0}(n), \ldots, \mu_{k-1}(n)\right)$, and $t^{*}=t(n)$ such that 


$$
\bar{y}_{n}\left(t^{*}\right)=\hat{y}_{n}\left(t^{*}\right)=\left.\frac{\partial \hat{y}_{n}\left(t^{*}\right)}{\partial t}\right|_{t=t^{*}}=\cdots=\left.\frac{\partial^{k} \hat{y}_{n}\left(t^{*}\right)}{\partial t^{k}}\right|_{t=t^{*}}=0,
$$

provided that $\left.\frac{\partial \hat{x}_{n}(t)}{\partial t}\right|_{t=t^{*}} \neq 0$. Indeed, $t^{*}$ corresponds to the point of a $k$-th order homoclinic tangency of $W^{u}\left(p_{1}\right)$ and $W^{s}\left(p_{1}\right)$, because $f_{\mu}^{N} \circ f^{n}\left(W_{l o c}^{u}\left(p_{1}\right)\right) \subset W^{u}\left(p_{1}\right)$.

In what follows we shall calculate approximately values of all $k+2$ parameters, including $t^{*}$, that satisfy (17). Now we start calculating parameter values $t^{*}, \mu_{0}(n), \mu_{1}(n)$, and so on. Step 1.

$$
\bar{y}_{n}(t)=\mu^{n}\left(a t^{2}+g(t)-\epsilon\right)+1 .
$$

Recall that $g(t)$ is $o\left(t^{2}\right)$ at $t=0$. Thus, $\bar{y}_{n}(t)=0$ for $t^{*} \approx \sqrt{a^{-1}\left(\epsilon-\mu^{n}\right)}$.

To simplify calculations notice that in a view of condition (6) $H_{2}\left(\lambda^{n}(1+t), 0\right) \sim \lambda^{2 n}$ for large $n$ 's. By Proposition 1 one can choose the saddle $p_{1}$ to be at least $(k-1)$-shrinking, so eigenvalues of $p_{1}$ satisfy $\lambda \mu^{k-1}=\tau<1$. We shall see that in this case we can choose $\epsilon$ in such a way that $\mu^{n} t^{*}(n) \rightarrow 0$ as $n \rightarrow \infty$ exponentially fast. Without loss of genericity we can choose the saddle $p_{1}$ so that $\lambda \mu^{k-1}<\tilde{\tau}<1$ (see Proposition (1).

Introduce some notations. $T_{n}=2 a \mu^{n} t^{*}, C_{s j}(t)=\partial^{j}\left(\left(\bar{y}_{n}(t)\right)^{s}\right) / \partial t^{j}$ for $s, j=1, \ldots, k$, $\partial_{r}^{j} H_{2}\left(x_{1}^{0}, x_{2}^{0}\right)=\partial^{j} H_{2}\left(x_{1}, x_{2}\right) /\left.\partial x_{r}^{j}\right|_{x_{1}=x_{1}^{0}, x_{2}=x_{2}^{0}}$.

In order to proceed we need the following

Proposition 6. There is a set of constants $\left\{C_{s j}\right\}_{j \leq s}, s, j=1, \ldots, k$ independent of $n$ such that

$$
C_{s j}\left(t^{*}(n)\right)=\left\{\begin{array}{l}
(1+o(1)) C_{s j} T_{n}^{j}\left(t^{*}\right)^{j-s} \quad \text { for } j \leq s \leq 2 j \\
(1+o(1)) C_{s j} T_{n}^{j}\left(t^{*}\right)^{-j} \text { for } s>2 j .
\end{array}\right.
$$

Proof of the Proposition: Recall that $\bar{y}_{n}\left(t^{*}\right)=0$. So, it is easy to see that $C_{j j}\left(t^{*}\right)=$ $\left[\mu^{n}\left(2 a t^{*}+g^{\prime}\left(t^{*}\right)\right)\right]^{j}=(1+o(1)) T_{n}^{j}$. For $s>j$ one can check that for some positive explicitly computable constant $C_{s j}$ we have $C_{s j}\left(t^{*}\right)=\left.\partial^{s-j} C_{j j}(t)\right|_{t=t^{*}}=(1+o(1)) C_{s j} T_{n}^{j}\left(t^{*}\right)^{j-s}$ for $j<s \leq 2 j$. Plugging in the value for $T_{n}$ we obtain $C_{(2 j) j}\left(t^{*}\right)=(1+o(1)) C_{(2 j) j} \mu^{j n}$. For $s>2 j C_{s j}\left(t^{*}\right)=(1+o(1)) C_{s j} T_{n}^{j}\left(t^{*}\right)^{-j}$. This completes the proof. Q.E.D.

Step 2 .

$$
\hat{y}_{n}\left(t^{*}\right)=\mu_{0}(n)+\sigma \lambda^{n}\left(1+t^{*}\right)+o\left(\lambda^{n}\right)=0 .
$$

Thus, $\mu_{0}(n) \approx-\sigma \lambda^{n}\left(1+t^{*}\right)$.

Notice that condition (6) implies $\partial_{2}^{j} H_{2}\left(\lambda^{n}\left(1+t^{*}\right), \bar{y}_{n}\left(t^{*}\right)\right) \sim \lambda^{n}$ for each $j=1, \ldots, k$. For some $C>0$

$$
\begin{array}{r}
\left.\left|\frac{\partial^{j}}{\partial t^{j}} H_{2}\left(\lambda^{n}(1+t), \bar{y}_{n}(t)\right)\right|_{t=t^{*}}|\leq| \sum_{s=0}^{j} \partial_{2}^{j-s} \partial_{1}^{s} H_{2}\left(\lambda^{n}(1+t), \bar{y}_{n}(t)\right)\right|_{t=t^{*}} \mid \leq \\
\leq\left|\sum_{s=0}^{j} \lambda^{n s} \partial_{2}^{j-s}\left(\partial_{1}^{s} H_{2}\right)\left(\lambda^{n}(1+t), \bar{y}_{n}(t)\right)\right|_{t=t^{*}} \mid \leq C \lambda^{n} \mu^{[j / 2] n},
\end{array}
$$

provided that $T_{n} \rightarrow 0$ as $n \rightarrow \infty$. The last inequality can be proven using formulas from Proposition 6 . 
Direct calculation shows that because of condition (16), $\bar{y}_{n}\left(t^{*}\right)=0$, and $T_{n} \rightarrow 0$ as $n \rightarrow \infty$ derivative $\partial_{t} H_{2}\left(\lambda^{n}\left(1+t^{*}\right), \bar{y}_{n}\left(t^{*}\right)\right)=o\left(\lambda^{n}\right)$.

Step 3.

$$
\left.\frac{\partial \hat{y}_{n}(t)}{\partial t}\right|_{t=t^{*}}=C_{11}\left(t^{*}\right) \mu_{1}+\sigma \lambda^{n}+o\left(\lambda^{n}\right)=0 .
$$

Therefore, $\mu_{1}(n) \approx-\sigma \lambda^{n} C_{11}^{-1}\left[T_{n}\right]^{-1}$.

Step 4.

$$
\left.\frac{\partial^{2} \hat{y}_{n}(t)}{\partial t^{2}}\right|_{t=t^{*}}=C_{22}\left(t^{*}\right) \mu_{2}(n)+(1+o(1)) C_{21} T_{n}\left(t^{*}\right)^{-1} \mu_{1}(n)+C \lambda^{n} \mu^{n}=0 .
$$

By Proposition 6, $C_{22}\left(t^{*}\right)=(1+o(1)) C_{22} T_{n}^{2}$. Now denote $D_{2}=C_{21} / C_{22}$. Thus, $\mu_{2}(n) \approx$ $-D_{2}\left[T_{n} t^{*}\right]^{-1} \mu_{1}(n)$.

Step 5.

$$
\begin{aligned}
\left.\frac{\partial^{3} \hat{y}_{n}(t)}{\partial t^{3}}\right|_{t=t^{*}}= & C_{33}\left(t^{*}\right) \mu_{3}(n)+(1+o(1)) C_{32} T_{n}^{2}\left(t^{*}\right)^{-1} \mu_{2}(n)+ \\
& +(1+o(1)) C_{31} T_{n}\left(t^{*}\right)^{-2} \mu_{1}(n)+C \lambda^{n} \mu^{n}=0 .
\end{aligned}
$$

By Proposition 6, $C_{33}\left(t^{*}\right)=(1+o(1)) C_{33} T_{n}^{3}$. Denote $D_{3}=\left(C_{32}+C_{31} C_{21}\right) / C_{33}$. Then using the formula for $C_{33}\left(t^{*}\right)$ we obtain $\mu_{3}(n) \approx-1 / C_{33}\left(C_{32}\left[T_{n} t^{*}\right]^{-1} \mu_{2}(n)-C_{31}\left[T_{n} t^{*}\right]^{-2} \mu_{1}(n)\right)$. Thus, $\mu_{3}(n) \approx-D_{3}\left[T_{n} t^{*}\right]^{-2} \mu_{1}(n)$.

Step $k+1$.

$$
\begin{aligned}
\left.\frac{\partial^{k-1} \hat{y}_{n}(t)}{\partial t^{k-1}}\right|_{t=t^{*}} & =C_{(k-1)(k-1)}\left(t^{*}\right) \mu_{k-1}+\mu_{0}(n)+C \lambda^{n} \mu^{[(k-1) / 2] n} \\
& \sum_{j=1}^{k-2}(1+o(1)) C_{(k-1)(k-j)} T_{n}^{k-j}\left(t^{*}\right)^{-j} \mu_{k-j}(n)=0 .
\end{aligned}
$$

By Proposition 6, we have $C_{(k-1)(k-1)}\left(t^{*}\right)=(1+o(1)) C_{(k-1)(k-1)} T_{n}^{k-1}$. Thus, $\mu_{k-1}(n) \approx$ $-1 / C_{(k-1)(k-1)} \sum_{j=0}^{k-2}(1+o(1)) C_{(k-1)(k-j-1)}\left[T_{n} t^{*}\right]^{-j} \mu_{k-j-1}(n)$. For some explicitly computable constant $D_{k-1}$, we obtain $\mu_{k-1}(n) \approx D_{k-1}\left[T_{n} t^{*}\right]^{-k+2} \mu_{1}(n)$.

At last

$$
\begin{array}{r}
\left.\frac{\partial^{k} \hat{y}_{n}(t)}{\partial t^{k}}\right|_{t=t^{*}}=C_{k k}\left(t^{*}\right) \gamma+\sum_{j=1}^{k-1}(1+o(1)) C_{k(k-j)} T_{n}^{k-j}\left(t^{*}\right)^{-j} \mu_{k-j}(n)+ \\
+\mu_{0}(n)+C \lambda^{n} \mu^{[k / 2] n}=0 .
\end{array}
$$

By Proposition $6 C_{k k}\left(t^{*}\right)=(1+o(1)) C_{k k} T_{n}^{k}$. Combining approximate equalities for $\mu_{j}(n) \approx$ $-D_{j}\left[T_{n} t^{*}\right]^{-j+1} \mu_{1}(n)$ we obtain $\gamma \approx-1 / C_{k k} \sum_{j} C_{k j}\left[T_{n} t^{*}\right]^{-j} \mu_{j}(n)=-D_{k}\left[T_{n} t^{*}\right]^{-k+1} \mu_{1}$ for some explicitly computable $D_{k}$. By a perturbation of the equation $y(x)=a x^{2}-g(x)$ we can guarantee that $D_{k}$ is different from 0 .

Let us plug in the equation for $\mu_{1}(n)$.

$$
\gamma \approx-\sigma D_{k} \lambda^{n} T_{n}^{-1}\left[T_{n} t^{*}\right]^{-k+1}=-(1+o(1)) \sigma D_{k}(2 a)^{-k} \lambda^{n} \mu^{-k n}\left(t^{*}\right)^{-2 k+1} .
$$

Now depending on the sing of $D_{k}$, we can choose the sign for $\sigma$ above so that both parts of this approximate equality have the same sign. By choosing an appropriate $\epsilon(n)$ we have 
the following the assymptotic formula $t^{*} \approx \sqrt{a^{-1}\left(\epsilon-\mu^{n}\right)} \approx\left(\lambda \mu^{-k}\right)^{n /(2 k-1)}$. Thus, we can satisfy assymptotic formula (26) for $\gamma$. Let us check the condition $\mu^{n} t^{*} \rightarrow 0$ exponentially fast. Since $p_{1}$ is at least $k-1$ shrinking $\left.\mu^{n} t^{*}=\left(\lambda \mu^{k-1}\right)\right)^{n}=\tau^{n}<1$. This complete the proof of Lemma 3. Q.E.D.

To complete the proof of Theorem 1 we apply Corollary 3 which allow a diffeomorphism with a $k$-th order tangency by a $C^{r}$-perturbation turn into a diffeomorphism with an arbitrarily high period $k$-degenerate orbit.

2.6. A Proof of Theorem 3. Fix a $C^{r}$ metric $\rho_{r}$ in $\operatorname{Diff}^{r}(M)$ defined by the standard way (see e.g. $[\mathrm{PM}]$ ). Let $f$ be a $C^{r}$ diffeomorphism which belongs to a Newhouse domain $\mathcal{N}$. Write $f \mapsto_{\varepsilon, r} g$ if $g$ is a $C^{r}$-perturbation of size at most $\varepsilon$ with respect to $\rho_{r}$. Consider an arbitrary sequence of positive integer numbers $\left\{a_{n}\right\}_{n=1}^{\infty}$.

Now for any $\varepsilon$ we construct a $3 \varepsilon$ perturbation $f_{3}$ of a diffeomorphism $f$ such that for some $n_{1}$ the diffeomorphism $f_{3}$ has $n_{1} a_{n_{1}}$ hyperbolic periodic orbits of period $n_{1}$. Hyperbolicity implies that the same is true for all diffeomorphisms sufficiently close to $f_{4}$.

Step 1. $f \mapsto_{\varepsilon, r} f_{1}$, where $f_{1}$ belongs to a Newhouse domain and is $C^{\infty}$ smooth.

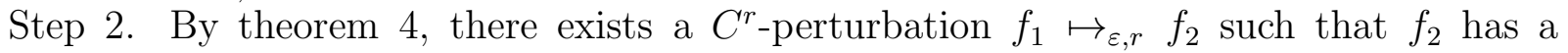
$k$-degenerate periodic orbit $q$ of an arbitrarily large period, where $k \geq r$.

Step 3. Let $n_{1}$ be a period of the $k$-degenerate periodic orbit $q$. It is easy to show that one can find $f_{2} \mapsto_{\varepsilon, r} f_{3}$ such that in a small neighborhood of $q f_{3}$ has $n_{1} a_{n_{1}}$ hyperbolic periodic points of period $n_{1}$.

Therefore, we show that an arbitrary $C^{r}$-close to $f$ there exist a neighborhood $U \subset$ $\operatorname{Diff}^{r}(M)$ with the following property for all $g \in U$

$$
\frac{\#\left\{x: g^{n}(x)=x\right\}}{a_{n}} \geq n .
$$

If the diffeomorphism $f_{1}$ belongs to a Newhouse domain $\mathcal{N} \subset \operatorname{Diff}^{r}(M)$, then we can choose perturbation in steps 1-3 so small that $f_{3}$ belongs to the same Newhouse domain $\mathcal{N}$. It is not difficult to see from steps 1-3 that for an open dense set in $\mathcal{N}$ the condition (27) holds at least for one $n$. Iterating steps 1-3 one contructs a residual set such that for each diffeomirphism $f$ from that residual set the condition (27) holds for an infinitely many $n$ 's. This completes the proof of Theorem 3 in the case $\operatorname{dim} M=2$. Q.E.D.

Note that similar inductive argument leads to the well-known Newhouse's phenomenon on infinitely many coexisting sinks $[\mathbb{N}],[\mathrm{PT}],[\mathbb{R}]$, and [TY].

Remark 3. No generic finite parameter family intersects a residual set $\mathcal{R}_{a}$ described in Theorem 目 with $a_{n}$ growing quckly enough, e.g. $a_{n}>n^{n}$. Indeed, it follows from the step 4 of previous section, where we take a highly degenerate orbit and perturb it. This operation is of large codimension and the larger step of our induction the more codimension. So, a member of $\mathcal{R}_{a}$ has to be in an arbitrary small neighborhood of a set of diffeomorphisms with an arbitrary high degenerate periodic orbit.

Remark 4. As we mentioned in the introduction, Theorem 9 implies Theorem 8 . 
2.7. A Proof of Theorem 3 in the case $\operatorname{dim} M>2$. We shall use the construction described in Step 1 of the proof of Theorem 3 .

Consider a compact manifold $M$ of dimension $\operatorname{dim} M>2$ and a diffeomorphism $F \in$ $\operatorname{Diff}^{r}(M)$. Fix a sequence of numbers $\left\{a_{n}\right\}_{n \in \mathbb{Z}_{+}}$. Suppose $F$ has a $C^{r}$-stable invariant twodimensional manifold $N \subset M$ and the restriction diffeomorphism $f=\left.F\right|_{N}: N \rightarrow N$ belongs to a Newhouse domain $\mathcal{N} \subset \operatorname{Diff}^{r}(N)$. $C^{r}$-stabililiby of the invariant manifold $N$ means that any $C^{r}$-perturbation $\tilde{F} \in \operatorname{Diff}^{r}(M)$ of $F$ also has a two-dimensional invariant manifold $\tilde{N}$ which is $C^{r}$-close to $N$ and induces a diffeomorphism $\tilde{f}=\left.\tilde{F}\right|_{N}: N \rightarrow N$ which is $C^{r}$-close to the restriction $f=\left.F\right|_{N}: N \rightarrow N$ (see Step 1 in 3.1 below for an exact formula of $\tilde{f}$ ). The Fenichel theorem $[\mathrm{Fe}]$ gives an explicit condition when $F$ has a $C^{r}$-stable invariant manifold. It is important that this is an open condition in $\operatorname{Diff}^{r}(M)$.

It is proved in the last subsection that the set of diffeomorphims for which the condition (27) is satisfied for at least one $n \in \mathbb{Z}_{+}$is open and dense in a Newhouse domain $\mathcal{N} \subset$ $\operatorname{Diff}^{r}(N)$. This implies that in a neighbourhood $U$ of $F$ in the space of diffeomorphisms $\operatorname{Diff}^{r}(M)$ there is an open and dense set $\mathcal{D}_{1}$ of diffeomorphisms such that each one satisfies the condition (27) for some $n \in \mathbb{Z}_{+}$.

Let $\mathcal{D}_{1, n_{1}}$ be an open subset of $\mathcal{D}_{1}$ consisting of diffeomorphisms for which the condition (27) holds (substituting $n=n_{1}$ ). There is an open and dense subset $D_{2, n_{1}, n_{2}}$ inside $\mathcal{D}_{1, n_{1}}$ of diffeomorphisms satisfying the condition (27) (substituting $n=n_{1}$ and $n=n_{2}$ with $n_{2}>n_{1}$ ). The union $D_{2}=\cup_{n_{2}>n_{1}} D_{2, n_{1}, n_{2}}$ is the open dense set inside $U$. Inductive application of these arguments shows that there exists a residual set inside of the open set $U$ the condition (27) holds for infinitely many of $n=n_{i}$ 's. This completes the proof of Theorem 3. Q.E.D.

\section{A Proof of Theorem 1}

The original proof of Artin-Mazur [AM] uses the fundamental theory of real algebraic approximations due to Nash. However, this method does not work for nonisolated periodic points (see pg.84 AM]). The method presented below is based on three basic tools: the Weierstrass approximation theorem, Elimination theory [Mu], and the Fenichel theorem [F]. The first two are well-known. The third is a deep fact about stability of invariant manifolds from dynamical systems theory. This method is advantageous in that it is simple and takes care of nonisolated (even nonhyperbolic) periodic points.

Let us start discribing the proof. Consider a $C^{r}$ diffeomorphism $f: M \rightarrow M$. We shall approximate $f$ by an A-M diffeomorphism with only hyperbolic periodic orbits. There are two steps:

3.1. Reduction to a problem for polynomial maps. Using the Whitney Embedding theorem embed $M$ into $\mathbb{R}^{N}$ for $N=2 \operatorname{dim} M+1$. Denote by $T$ a tube neighborhood of $M$. For any fixed $r \in \mathbb{Z}_{+}$one can extend $f: M \rightarrow M$ to a diffeomorphism $F: T \rightarrow T$ such that $F$ restricted to $M$ coincides with $f$ and if $F$ contracts along transversal to $M$ directions sufficiently strongly, then by the Fenichel theorem $[\mathrm{Fe}$ each diffeomorphism $\tilde{F}: T \rightarrow T$ which is $C^{r}$-close to $F$ has a $C^{r}$ smooth invariant manifold $\tilde{M}$ which is $C^{r}$-close to $M$. Denote by $\pi: \tilde{M} \rightarrow M$ a diffeomorphism from $\tilde{M}$ to $M$ which can be obtained by projection along the normal to $M$ directions. Then $\tilde{f}=\left.\pi^{-1} \circ \tilde{F}\right|_{\tilde{M}} \circ \pi: M \rightarrow M$ is a diffeomorphism which is $C^{r}$-close to $f$. By the Weierstrass approximation theorem one can approximate a diffeomorphism $F: T \rightarrow T$ of an open set $T$ in the Euclidean space $\mathbb{R}^{N}$ into itself by a polynomial diffeomorphism $\tilde{F}=\left.P\right|_{T}: T \rightarrow T$. Notice that if $\tilde{F}$ has only hyperbolic periodic 
orbits, then the induced diffeomorphism $\tilde{f}=\left.\pi^{-1} \circ \tilde{F}\right|_{\tilde{M}} \circ \pi: M \rightarrow M$ also has only hyperbolic periodic orbits.

We shall prove that, indeed, one can approximate any diffeomorphism $F: T \rightarrow T$ by a polynomial diffeomorphism $\tilde{F}=\left.P\right|_{T}: T \rightarrow T$ which has only hyperbolic periodic orbits.

Let $D \in \mathbb{Z}_{+}$. Denote by $A_{N}^{D}$ the space of vector-polynomials $P: \mathbb{R}^{N} \rightarrow \mathbb{R}^{N}$ of degree at most $D$. If $\mu=\mu(N, D)=\#\left\{\alpha \in \mathbb{Z}_{+}^{N}:|\alpha| \leq D\right\}$, then $A_{N}^{D}$ is isomorphic to $\mathbb{R}^{\mu}$. Consider $A_{N}^{D}$ with the Lebesgue measure on it. In what follows we call a vector-polynomial by a polynomial for brevity.

Step 2. For any $D \in \mathbb{Z}_{+}$an almost every polynomial $P: \mathbb{R}^{N} \rightarrow \mathbb{R}^{N}$ from $A_{N}^{D}$ has only hyperbolic periodic orbits and their number grows at most exponentially.

The second part of this statement is easy provided that the first is true. Indeed, fix $k \in \mathbb{Z}_{+}, k>0$ and consider the system

$$
P\left(x_{1}\right)-x_{2}=0, P\left(x_{2}\right)-x_{3}=0, \ldots, P\left(x_{k}\right)-x_{1}=0 .
$$

This system has $N k$ equations, each of them of degree at most $D$. By the Bezout theorem the number of isolated solutions is at most $D^{k N} \leq\left(D^{N}\right)^{k}$. If all periodic points are hyperbolic, then they are all isolated and this completes the proof.

Fix $k \in \mathbb{Z}_{+}, k>0$. Let $\alpha=\left(\alpha_{1}, \ldots, \alpha_{N}\right) \in \mathbb{Z}_{+}^{N}$ be a multiindex, $|\alpha|=\sum_{i} \alpha_{i}$. Fix a coordinate system in $\mathbb{R}^{N}$ so one can write each polynomial $P(a, \cdot): \mathbb{R}^{N} \rightarrow \mathbb{R}^{N}$ from $A_{N}^{D}$ in the form

$$
\begin{aligned}
& \quad P(a, x)=\sum_{|\alpha| \leq D} a_{\alpha} x^{\alpha}, \text { where } a=\left(\left\{a_{\alpha}\right\}_{|\alpha| \leq D}\right) \in \mathbb{R}^{\mu}, \\
& x=\left(x_{1}, \ldots, x_{N}\right) \in \mathbb{R}^{N}, \text { and } x^{\alpha}=x_{1}^{\alpha_{1}} \ldots x_{N}^{\alpha_{N}} .
\end{aligned}
$$

Lemma 4. Let $\lambda_{0} \in \mathbb{C}$ and $\left|\lambda_{0}\right|=1$. For any $D \in \mathbb{Z}_{+}$an almost every polynomial $P$ : $\mathbb{R}^{N} \rightarrow \mathbb{R}^{N}$ from $A_{N}^{D}$ has no periodic orbits with the eigenvalue $\lambda_{0}$.

Denote $P^{(k)}(a, \cdot)=P(a, \cdot) \circ \cdots \circ P(a, \cdot): \mathbb{R}^{N} \rightarrow \mathbb{R}^{N}$ ( $k$ times), the linearization matrix of the map $P^{(k)}(a, \cdot)$ at a point $x$ by $d_{x}\left(P^{(k)}\right)(a, x)$, and the $N \times N$ identity matrix by $I d$. Let $\lambda \in \mathbb{C}$ be a complex number. Denote $D(a, \lambda, x)=\operatorname{det}\left(d_{x}\left(P^{(k)}\right)(a, x)-\lambda I d\right)$. Every periodic orbit of period $k$, which has an eigenvalue $\lambda$, satisfies the following system:

$$
\left\{\begin{array}{l}
P^{(k)}(a, x)-x=0, \quad x=\left(x_{1}, \ldots x_{N}\right) \in \mathbb{R}^{N} \\
D(a, \lambda, x)=0, \quad a \in \mathbb{R}^{\mu}
\end{array}\right.
$$

The general goal is to prove that for a "generic" choice of coefficients $a \in \mathbb{R}^{\mu}$ of $P(a, \cdot)$ this system has no solutions satisfying the condition $|\lambda|=1$ or there is no nonhyperbolic periodic orbit of period $k$. First, we prove that a "generic" choice of coefficients $a \in \mathbb{R}^{\mu}$ of $P(a, \cdot)$ has no periodic points with the eigenvalue $\lambda=\lambda_{0}$.

Notice that the system (29) including the condition $\lambda=\lambda_{0}($ or $|\lambda|=1)$ consists of $N+2$ equations and for each value $a$ only $N+1$ indetermine variables $x_{1}, \ldots, x_{N}, \lambda$. It might be clear intuitively that for a "generic" $a \in \mathbb{R}^{\mu}$ there is no solution, because the number of equations is more than the number of indeterminates. To prove it rigorously for $\lambda=\lambda_{0}$ we shall apply Elimination theory. 
3.2. Elimination theory. Let $\mathbb{C}^{m}$ denote the $m$-dimensional complex space $z=\left(z_{1}, \ldots, z_{m}\right) \in$ $\mathbb{C}^{m}, \quad m \in \mathbb{Z}_{+}$. A set $V$ in $\mathbb{C}^{m}$ is called a closed algebraic set in $\mathbb{C}^{m}$ if there is a finite set of polynomials $F_{1}, \ldots F_{s}$ in $z_{1}, \ldots, z_{m}$ such that

$$
V\left(F_{1}, \ldots, F_{s}\right)=\left\{\left(z_{1}, \ldots, z_{m}\right) \in \mathbb{C}^{m} \mid F_{j}\left(z_{1}, \ldots, z_{m}\right)=0,1 \leq j \leq s\right\} .
$$

One can define a topology in $\mathbb{C}^{m}$, called the Zariski topology, whose closed sets are closed algebraic sets in $\mathbb{C}^{m}$. This, indeed, defines a topology, because the set of closed algebraic sets is closed under a finite union and an arbitrary intersection. Sometimes, closed algebraic sets are also called Zariski closed sets.

Definition 5. A subset $S$ of $\mathbb{C}^{m}$ is called constructible if it is in the Boolean algebra generated by the closed algebraic sets; or equivalently if $S$ is a disjoint union $T_{1} \cup \cdots \cup T_{k}$, where $T_{i}$ is locally closed, i.e. $T_{i}=T_{i}^{\prime}-T_{i}^{\prime \prime}, T_{i}^{\prime}-$ a closed algebraic set and $T_{i}^{\prime \prime} \subset T_{i}^{\prime}-$ a smaller closed algebraic.

One of the main results of Elimination theory is the following

Theorem 5. $\mathrm{Mu}$ Let $V \subset \mathbb{C}^{\mu} \times \mathbb{C}^{N}$ be a constructible set and $\pi: \mathbb{C}^{\mu} \times \mathbb{C}^{N} \rightarrow \mathbb{C}^{\mu}$ be the natural projection. Then $\pi(V) \subset \mathbb{C}^{\mu}$ is a constructible set.

Remark 5. An elementary description of elimination theory can be found in books Jacobson [J] and van der Waerden [W].

3.3. Proof of Lemma 4 or application of Elimination theory to the system (29). Put $\lambda=\lambda_{0}$ and consider the system (29) as if it is defined for $(a ; x) \in \mathbb{C}^{\mu} \times \mathbb{C}^{N}$. Then it defines a closed algebraic set $V_{k}\left(\lambda_{0}\right) \subset \mathbb{C}^{\mu} \times \mathbb{C}^{N}$. By Theorem 5 the natural projection $\pi: \mathbb{C}^{\mu} \times \mathbb{C}^{N} \rightarrow \mathbb{C}^{\mu}$ of $V_{k}\left(\lambda_{0}\right)$, namely $\pi\left(V_{k}\left(\lambda_{0}\right)\right) \subset \mathbb{C}^{\mu}$, is a constructible set. The only thing left to show is that $\pi\left(V_{k}\left(\lambda_{0}\right)\right) \neq \mathbb{C}^{\mu}$ and has a positive codimension. Recall that $\left|\lambda_{0}\right|=1$ and $\mathbb{C}^{\mu}$ is the space of coefficients of polynomial of degree $D$.

Proposition 7. Let $\mathbb{R}^{\mu}$ be naturally embedded into $\mathbb{C}^{\mu}$. Then there is an open set $U \subset \mathbb{C}^{\mu}$ such that $U \cap \mathbb{R}^{\mu} \neq \emptyset$ and for any $a \in U$ the corresponding polynomial $P(a, \cdot): \mathbb{C}^{N} \rightarrow \mathbb{C}^{N}$ of degree has exactly $D^{N k}$ periodic points of period $k$ and all of them are hyperbolic.

Proof: Consider the homogeneous polynomial $P\left(a^{*}, \cdot\right): \mathbb{C}^{N} \rightarrow \mathbb{C}^{N}$ of degree $D P\left(a^{*}, \cdot\right)$ : $\left(z_{1}, \ldots, z_{N}\right) \mapsto\left(z_{1}^{D} \ldots, z_{N}^{D}\right)$. It is easy to see that $P$ has exactly $D^{N k}$ periodic points of period $k$ all of which are hyperbolic. From one side hyperbolicity of periodic points of period $k$ of $P$ implies that any polynomial mapping $\tilde{P}$, which is a small perturbation of $P$, has at least $D^{N k}$ hyperbolic points of period $k$, but from the other side Bezout's Theorem implies that a polynomial of degree $D$ has at most $D^{N k}$ periodic point of period $k$. Thus, there is a neighborhood $U \subset \mathbb{C}^{\mu}$ of $a^{*}$ such that for any $a \in U$ the corresponding polynomial $P(a, \cdot)$ : $\mathbb{C}^{N} \rightarrow \mathbb{C}^{N}$ has only hyperbolic periodic points of period $k$ and by definition $U \cap \pi\left(V_{k}\left(\lambda_{0}\right)\right)=\emptyset$. Since, $\pi\left(V_{k}\left(\lambda_{0}\right)\right)$ is constructible, this implies that $\pi\left(V_{k}\left(\lambda_{0}\right)\right)$ has positive codimension in $\mathbb{C}^{\mu}$. This completes the proof of Proposition.

By Proposition 7 the restriction $\pi\left(V_{k}\left(\lambda_{0}\right)\right) \cap \mathbb{R}^{\mu}$ has positive codimension and, therefore, measure zero in $\mathbb{R}^{\mu}$. Thus, almost every polynomial $P(a, \cdot)$ from $A_{N}^{D}=\mathbb{R}^{\mu}$ has no periodic points of period $k$ with the eigenvalue $\lambda_{0}$. Intersection over all $k \in \mathbb{Z}_{+}$gives that the same is true for all periods. This completes the proof of Lemma 4 Q.E.D. 
3.4. Completion of Proof of Step 2 of Theorem 1. Consider the system (29) as if it is defined for $(a, \lambda ; x) \in \mathbb{C}^{\mu} \times \mathbb{C} \times \mathbb{C}^{N}$. It defines a closed algebraic set, denoted by $V_{k} \subset \mathbb{C}^{\mu} \times \mathbb{C} \times \mathbb{C}^{N}$. By Theorem 5 the natural projection $\pi: \mathbb{C}^{\mu} \times \mathbb{C} \times \mathbb{C}^{N} \rightarrow \mathbb{C}^{\mu} \times \mathbb{C}$ of $V_{k}$, namely, $S_{k}=\pi\left(V_{k}\right) \subset \mathbb{C}^{\mu} \times \mathbb{C}$, is a constructible set.

Consider natural projections $\pi_{1}: \mathbb{C}^{\mu} \times \mathbb{C} \rightarrow \mathbb{C}^{\mu}$ and $\pi_{2}: \mathbb{C}^{\mu} \times \mathbb{C} \rightarrow \mathbb{C}$. It follows from Proposition 7 that $S_{k}$ has dimension $\mu$. Indeed, the projection $\pi_{1}\left(S_{k}\right)=W_{k}$ contains an open set $U \subset \mathbb{C}^{\mu}$ and $S_{k}$ does not intersect a neighborhood of $U \times\{\lambda:|\lambda|=1\} \subset \mathbb{C}^{\mu} \times \mathbb{C}$.

By Theorem 5 that $\pi_{1}\left(S_{k}\right)=W_{k}$ is constructible and by has Proposition $7 \operatorname{dim} W_{k}=\mu$. By Sard's lemma for algebraic sets [Mu there exists a proper algebraic set $\Sigma_{k} \subset S_{k}$ such that $\tilde{S}_{k}=S_{k} \backslash \Sigma$ outside $\Sigma_{k}$ the restricted to $\tilde{S}_{k}$ map $p_{k}=\left.\pi_{1}\right|_{\tilde{S}_{k}}: \tilde{S}_{k} \rightarrow \mathbb{C}^{\mu}$ has no critical points. Thus, outside of some proper closed algebraic set $\Sigma_{k}^{\prime} \subset \mathbb{C}^{\mu}$ the map $p_{k}: \tilde{S}_{k} \rightarrow \mathbb{C}^{\mu} \backslash \Sigma_{k}^{\prime}$ is locally invertible.

Recall that our goal is to show that $Z=\pi_{1}\left(S_{k} \cap\{\lambda:|\lambda|=1\}\right) \cap \mathbb{R}^{\mu}$ has measure zero in $A_{N}^{D}=\mathbb{R}^{\mu}$. It is sufficient to show that this locally.

Let $a \in \mathbb{R}^{\mu} \backslash \Sigma_{k}^{\prime}$ and $U \subset \mathbb{C}^{\mu} \backslash \Sigma_{k}^{\prime}$ be a neighborhood of $a$. By construction the map $p_{1}: \tilde{S}_{k} \rightarrow \mathbb{C}^{\mu} \backslash \Sigma_{k}^{\prime}$ is locally invertible, so the preimage $p_{1}^{-1}(U)$ consists of a finite disjoint union of open sets $\cup_{j \in J} U_{j} \subset S_{k}$. Thus, one can define a finite collection of analytic functions $\left\{\lambda_{k, j}=\pi_{2} \circ p_{k, j}^{-1}: U \rightarrow \mathbb{C}\right\}_{j \in J}$, where $p_{k, j}^{-1}: U \rightarrow U_{j}$ is the inverse of the restriction $\left.p_{k}\right|_{U_{j}}: U_{j} \rightarrow U$. We need to show that

$$
\cup_{j \in J}\left\{\lambda_{k, j}^{-1}(\{\lambda:|\lambda|=1\}) \cap \mathbb{R}^{\mu}\right.
$$

has measure zero. If for some $j \in J$ the function $\lambda_{k, j}: U \rightarrow \mathbb{C}$ is equal to a constant $\lambda$, then by Proposition $\square$ we have $|\lambda| \neq 1$ and the preimage $\lambda_{j}^{-1}(Z)=\emptyset$. If for some $j \in J$ the function $\lambda_{k, j}: U \rightarrow \mathbb{C}$ is not constant, then the set $\lambda_{j}^{-1}(\{\lambda:|\lambda|=1\}) \cap \mathbb{R}^{\mu}$ is real analytic set. Moreover, it follows from Proposition 7 and the identity theorem that $\lambda_{j}^{-1}(\{\lambda:|\lambda|=1\}) \cap \mathbb{R}^{\mu}$ has to be nowhere dense and, therefore, have a positive codimension. A real analytic set of positive codimension has measure zero. It follows e.g. from the fact that a real analytic can be stratified (see e.g. [H] or [GM]), i.e., in particular, can be decomposed into at most countable union of semianalytic manifolds. Each semianalytic manifold must have a positive codimension and, therefore, measure zero. This implies that for almost every $a \in \mathbb{R}^{\mu}$ the system (29) has no solutions for $|\lambda|=1$. Intersection of all periods $k \in \mathbb{Z}_{+}$completes the proof of step 2 .

Let us complete the proof of Theorem 11. Application of Step 1 shows that a diffeomorphism $f: M \rightarrow M$ can be extended to a tube neighborhood $T$ of $M F: T \rightarrow T$ and that it is sufficient to approximate $F$ by a diffeomorphism $\tilde{F}: T \rightarrow T$ which has only hyperbolic periodic points. By the Weierstrass approximation theorem $F$ can be approximated by a polynomial diffeomorphism $\tilde{F}=\left.P\right|_{T}: T \rightarrow T$. Since, in the space of polynomial of any degree $D$ polynomial maps with only hyperbolic periodic points form a full measure set one can choose $\tilde{F}=\left.P\right|_{T}: T \rightarrow T$ which has only hyperbolic periodic points. If $\tilde{F}: T \rightarrow T$ has only hyperbolic periodic points, then its restriction to an invariant manifold also has only hyperbolic periodic points. This completes the proof of Theorem 11.

Remark 6. In order to give a positive answer to the Artin-Mazur question stated in the introduction it is sufficient to use only Step 1 and Lemma 4 of the above proof. 


\section{Prevalence and open problems}

There are two point of view on a notion of genericity in dynamical systems, singularity theory and etc.: topological and metrical. Topological genericity which goes back to Baire is standard and widely accepted. It says that a property of dynamical systems is generic if systems with that property form a residual set.

However, it is easy to construct a residual set in the segment $[0,1]$ which has measure zero. Different examples from the KAM theory, small denominators, fractal geometry, and so on show that topological description is not always a good one (see [HSY] for more examples).

Let us describe another point of view which goes back to Kolmogorov. In his plenary talk on the International Mathematical Congress in 1954, A.N. Kolmogorov proposed to judge whether a phenomenon is generic or not by considering a generic finite parameter family with the Lebesgue measure on a parameter space and looking at measure of parameters corresponding to that phenomenon. In $[\mathbb{K}]$ the author proposed the following definition:

Let $B^{n}$ be an $n$-dimensional ball. Denote by $\operatorname{Diff}^{r}\left(M, B^{n}\right)$ the space of $n$-parameters families of diffeomorphisms $\left\{f_{\varepsilon}\right\}_{\varepsilon \in B^{n}}$ with the uniform $C^{k}$ topology.

Definition 6. We call a set $P \subset \operatorname{Diff}^{r}(M)$ an $n$-prevalent with respect to an $n$-parameter family $\left\{f_{\varepsilon}\right\}_{\varepsilon \in B^{n}}$ if $P$ restricted to that family form a set of full-measure with respect to the natural Lebesgue measure in the space of parameters:

$$
\operatorname{mes}\left\{\varepsilon \in B^{n}: f_{\varepsilon} \in P\right\}-\text { full-measure. }
$$

We call a set $P \subset \operatorname{Diff}^{r}(M)$ a strictly $n$-prevalent if the following two conditions hold:

A) $P$ is prevalent with respect to an open dense set of $n$-parameter families $\left\{f_{\varepsilon}\right\}_{\varepsilon \in B^{n}} \in$ $\operatorname{Diff}^{r}\left(M, B^{n}\right)$;

$B)$ For any element $f \in \operatorname{Diff}^{r}(M)$ there exists an $n$-parameter family of diffeomorphisms $\left\{f_{\varepsilon}\right\}_{\varepsilon \in B^{n}} \in \operatorname{Diff}^{r}\left(M, B^{n}\right)$ which passes through $f$, i.e. $f=f_{0}$ and $P$ is prevalent with respect to $\left\{f_{\varepsilon}\right\}_{\varepsilon \in B^{n}}$.

$A$ set $P \subset \operatorname{Diff}^{r}(M)$ is called n-prevalent if it contains a countable intersection of $n$ prevalent sets. We also call a set prevalent if it is $n$-prevalent for some $n$ and neglectable if the complement is prevalent.

It easily follows from the definition that a countable intersection of $n$-prevalent sets is $n$-prevalent.

Proposition 8. [K] If $P \subset \mathbb{R}^{N}$ and $P$ is n-prevalent for some $n<N$, then $P$ has full measure in $\mathbb{R}^{N}$.

It shows that on the contrary to the topological genericity test of this definition in a finite-dimensional case gives a satisfactory result.

In [K] it is proven that certain fundamental facts from the singularity theory and the theory of dynamical systems such as transversality theorems, the Whitney embedding, the Mather stability, and the Kupka-Smale theorems, which are topologically generic, are also prevalent. In [HSY] a definition of prevalent set in an infinite dimensional linear space is proposed.

In a view of this it is natural to pose following problems:

Problem 1. Do Artin-Mazur diffeomorphisms form a prevalent set? 
Problem 2. Whether or not Newhouse's phenomenon on infinitely many coexisting sinks 1-prevalent?

Recall also a growth problem for vector fields from Artin-Mazur's paper [AM]:

Problem 3. Let $X$ be a differentiable vector field on a compact manifold $M$. Denote by $N_{t}(X)$ the number of periodic orbits of $X$, period less than or equal to $t$. Does $N_{t}(X)$ grow at most exponentially for some reasonable dense class of vector fields?

Acknowledgments: I would like to express my warmest thanks to my thesis advisor John Mather. He proposed to look at the problem of growth of number of periodic orbits and suggested the pertinent idea that a highly degenerate periodic orbit can generate a lot of periodic orbits in an open way. Several discussions of elimination theory with him were fruitful for me. J. Milnor gave the reference to the book of Bowen and proposed the excellent title. A moral support of J.Milnor was very important for me. A.Katok pointed out to me the question posed by Artin - Mazur (see Theorem 1). Remarks of D. Dolgopiat, Yu. Ilyashenko, J. Mather, J. Milnor, S. Patinkin, and G. Yuan on the text are highly appreciated. G. Levin mentioned to me the elimination theory. Let me express sincere gratitude to all of them.

\section{REFERENCES}

[AM] M. Artin, B. Mazur, Periodic orbits, Annals of Mathematics, 81, 1965, 82-99

[Ba] V. Baladi, Dynamical zeta functions, Series C: Mathematical and Physical Sciences, vol. 464 in Real and Complex dynamical systems, edited by B.Branner \& P.Hjorth

[BC] M. Benedicks, L. Carleson, Dynamics of the Henon map, Annals of Mathematics 133 (1991) 73-169

[BD] C. Bonatti, Z. Diaz, Connexions hétéroclines ae généricité d'une infinité ou se sources, prepublications du laboraroire de topologie, Dijon

[Bo] R. Bowen, On Axiom A diffeomorphisms, Regional Conference Series in Mathematics, no. 35, AMS, Providence, RI, 1978

[Fe] N. Fenichel, Persistence and smoothness of invariant manifolds for flows, Indiana Univ. Math. J. 21 (1971) 193-226

[GST] S. V. Gonchenko, L. P. Shil'nikov, and D. V. Tuvaev, On models with non-rough Poincaré homoclinic curves, Physica D 62 (1993), 1-14

[GM] M. Goresky, R. MacPherson, Stratified Morse Theory, A Series of Modern Surveys in Mathematics, Springer-Verlag, 1980

[H] R. Hardt, Topological properties of subanalytic sets, Trans. Amer. Math. Soc. 211 (1975) 57-70.

[HK] B. Hasselblatt, A. Katok, Introduction to the modern theory of dynamical systems. Encyclopedia of Mathematics and its Applications, 54. Cambridge University Press, Cambridge, 1995.

[HSY] B. R. Hunt, T. Sauer, and J. A. Yorke, Prevalence: a translation-invariant almost every for infinite dimensional spaces, Bull. Amer. Math. Soc. 27 (1992), 217-238; Prevalence: an addendum, Bull. Amer. Math. Soc. 28 (1993), 306-307.

[IY] Yu. S. Ilyashenko, S. Yu. Yakovenko, Finitely-Smooth normal forms for local families of diffeomorphisms and vector fields, Russ. Math. Survey (46) 1991, no.1, 1-43

[J] N. Jacobson, Basic Algebra, vol.1, 1974

$[\mathrm{K}] \quad$ V. Yu. Kaloshin, Some prevalent properties of smooth dynamical systems, in Differential equations with complex and real time, ed. Yu. S. Il'yashenko, Proc. Steklov Inst. Math. 213 (1996).

[Mu] D. Mumford, Algebraic Geometry I, Complex Projective Varieties, Springer-Verlag, New York, 1976

[MV] L. Mora, M. Viana, Abundance of strange attractors, Acta Math. 171, 1993, 1-71

[N] S. Newhouse, Diffeomorphisms with infinitely many sinks, Topology 13 (1974), 9-18

[PM] J. Palis, V. de Melo, Geometric Theory of Dynamical Systems, Springer-Verlag, 1982

[PT] J. Palis, \& F. Takens, Hyperbolicity \& sensitive chaotic dynamics at homoclinic bifurcations, Cambridge University Press 1993.

\footnotetext{
${ }^{3} \mathrm{~A}$ partial answer is in [TY].
} 
[PS] E. Pujals, M. Sambarino, Homoclinic tangencies and hyperbolicity for surface diffeomorphisms: A conjecture of Palis, preprint, 1998

[R] C. Robinson, Bifurcation to infinitely many sinks, Comm. in Math. Physics, 106, 433-459, 1983

[RG] E. Rozales-Gorsalez, Growth of periodic orbits of dynamical systems, Func. Anal. Appl. 25 (4) (1991), 254-262

[S] S. Smale, Differentiable Dynamical Systems, Bull. Amer. Math. Soc. 73 (1967), 747-817.

[Si] C. Simon, Instability of $\operatorname{Diff}^{k}\left(T^{3}\right)$ and the nondegenericity of rational zeta functions, Transc. of Amer. Math. Soc. vol.174 (1973), 217-242

[TY] L. Tedeschini-Lalli \& J. A. Yorke, Infinitely many coexisting sinks, Comm. in Math. Physics, 106, 1986, 635-657

[W] B. L. van der Waerden, Modern Algebra, vol.II, Frederick Ungar Publishing Co. New York, 1950

Department of Mathematics, Princeton University, Princeton NJ 08544-1000

E-mail address: kaloshin@math.princeton.edu 